\title{
The brown dwarf atmosphere monitoring (BAM) project
}

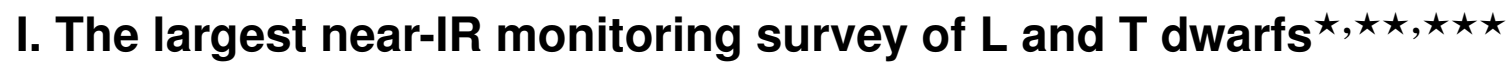

\author{
P. A. Wilson ${ }^{1}$, A. Rajan ${ }^{2}$, and J. Patience ${ }^{1,2}$ \\ 1 Astrophysics Group, School of Physics, University of Exeter, Stocker Road, Exeter EX4 4QL, UK \\ e-mail: paw@astro.ex.ac.uk \\ 2 School of Earth \& Space Exploration, Arizona State University, Tempe AZ 8528, USA
}

Received 6 November 2013 / accepted 17 April 2014

\section{ABSTRACT}

\begin{abstract}
Using the SofI instrument on the $3.5 \mathrm{~m}$ New Technology Telescope, we have conducted an extensive near-infrared monitoring survey of an unbiased sample of 69 brown dwarfs spanning the L0 to T8 spectral range, with at least one example of each spectral type. Each target was observed for a $2-4 \mathrm{~h}$ period in the $J_{\mathrm{s}}$-band, and the median photometric precision of the data is $\sim 0.7 \%$. A total of 14 brown dwarfs were identified as variables with min-to-max amplitudes ranging from $1.7 \%$ to $10.8 \%$ over the observed duration. All variables satisfy a statistical significance threshold with a $p$-value $\leq 5 \%$ based on comparison with a median reference star light curve. Approximately half of the variables show pure sinusoidal amplitude variations similar to 2MASSJ2139+0220, and the remainder show multi-component variability in their light curves similar to SIMPJ0136+0933. It has been suggested that the L-T transition should be a region of a higher degree of variability if patchy clouds are present, and this survey was designed to test the patchy cloud model with photometric monitoring of both the L-T transition and non-transition brown dwarfs. The measured frequency of variables is $13_{-4}^{+10} \%$ across the L7-T4 spectral range, indistinguishable from the frequency of variables of the earlier spectral types $\left(30_{-8}^{+11 \%}\right)$, the later spectral types $\left(13_{-4}^{+10} \%\right)$, or the combination of all non-transition region brown dwarfs $\left(22_{-5}^{+7} \%\right)$. The variables are not concentrated in the transition, in a specific colour, or in binary systems. Of the brown dwarfs previously monitored for variability, only $\sim 60 \%$ maintained the state of variability (variable or constant), with the remaining switching states. The 14 variables include 9 newly identified variables that will provide important systems for follow-up multi-wavelength monitoring to further investigate brown dwarf atmosphere physics.
\end{abstract}

Key words. techniques: photometric - brown dwarfs - stars: low-mass - stars: atmospheres

\section{Introduction}

The L, T, and Y-type brown dwarfs represent a link between the coolest stars and giant planets. Many brown dwarfs are even cooler than currently observable exoplanetary atmospheres (e.g. HR 8799b, HD 189733b; Barman et al. 2011; Sing et al. 2009, 2011). The recently discovered Y dwarfs (Cushing et al. 2011) approach the temperature of Jupiter. Since brown dwarfs never achieve a stable nuclear burning phase, they cool throughout their lifetimes, and temperature, rather than mass, is the dominant factor in defining the spectral sequence. As they cool, their atmospheres undergo changes in the chemistry and physical processes that sculpt their emergent spectra. While spectroscopy can be used to investigate atmospheric constituents and chemistry, photometric monitoring is an effective means to search for evidence of surface brightness inhomogeneities caused by cloud features, storms, or activity.

The transition region from late-L to early- $\mathrm{T}$ encompasses a particularly interesting change in physical properties, as the atmospheres transform from dusty to clear over a narrow effective

\footnotetext{
* Based on observations made with ESO Telescopes at La Silla Observatory under programme ID 188.C-0493.

$\star \star$ Tables 1,2 , and 4 are available in electronic form at http: //www . aanda.org

$\star \star \star$ Light curves (target and references) are only available at the CDS via anonymous ftp to cdsarc.u-strasbg. fr (130.79.128.5) or via http://cdsarc.u-strasbg.fr/viz-bin/qcat?J/A+A/566/A111
}

temperature range, and the observed infrared colours reverse from red to blue. This is predicted to be an effect of the formation and eventual dissipation of dusty clouds in brown dwarf atmospheres (Chabrier \& Baraffe 2000; Marley et al. 2002; Burrows et al. 2006). Broadly speaking, as brown dwarfs cool through the spectral sequence, the lower temperatures allow more complex molecules to form, resulting in condensate clouds. When the temperature is cool enough, large condensate grains cannot remain suspended high in the atmosphere and sink below the observable photosphere, allowing methane and molecular hydrogen to become the dominant absorbers. Although there are several existing models for condensate cloud evolution, most cannot easily explain the rapid colour change from red to blue over the L-to-T transition. A systematic survey of variability in brown dwarfs including both L-T transition objects and comparison hotter/cooler objects is required to search for differences in the structure of condensate clouds in this important regime.

Existing photometric monitoring campaigns of brown dwarfs have been conducted at different wavelengths: optical bands (e.g. Tinney \& Tolley 1999; Koen 2013), near-IR bands (e.g. Artigau et al. 2003; Khandrika et al. 2013; Buenzli et al. 2014), mid-IR (e.g. Morales-Calderón et al. 2006), and radio frequencies (e.g. Berger 2006). From small ( $<20$ objects) initial samples of ultracool field dwarfs, frequencies of variables ranged from $0 \%$ to $100 \%$ (e.g. summary in Bailer-Jones 2005), and results from larger studies ( 25 objects) have measured the frequency of variables to be in the range of $20 \%$ to $30 \%$ (e.g. 
Khandrika et al. 2013; Buenzli et al. 2014). Examples of objects that vary in multiple wavebands have been identified (e.g. 2MASS J22282889-4310262 Clarke et al. 2008; Buenzli et al. 2012, SIMP J013656.5+093347.3 Artigau et al. 2009, 2MASS J21392676+0220226 Radigan et al. 2012), as well as objects recorded as variable in one wavelength range, but not another (e.g. 2MASS J15344984-2952274, Koen et al. 2004). A small set of variable sources have been monitored contemporaneously at multiple wavelengths, with the combined results being used to infer the vertical extent of atmospheric features and to investigate atmospheric circulation patterns (e.g. Buenzli et al. 2012). Given the unique probe of the atmospheric structure that multi-wavelength observations provide, it is essential to identify a larger set of known variables across a broad range of effective temperatures.

Most monitoring programs have involved observation sequences spanning a few hours, but some studies have searched for longer timescale variations (e.g. Gelino et al. 2002; Enoch et al. 2003). A timescale of a few hours is well matched to a search for rotation-modulated variability, since expected rotation periods are $\sim 2-12 \mathrm{~h}$ for $\mathrm{L}$ and $\mathrm{T}$ dwarfs, considering the range of measured $v \sin i$ values $\left(10-60 \mathrm{~km} \mathrm{~s}^{-1}\right.$ for L dwarfs and $15-40 \mathrm{~km} \mathrm{~s}^{-1}$ for $\mathrm{T}$ dwarfs; Zapatero Osorio et al. 2006) and the $\sim 0.08-0.10 M_{\odot}$ radius of these objects from evolutionary models at the age of the field (Baraffe et al. 2003). Periodogram analysis of some variables has shown clear peaks associated with periods in the range of $\sim 2-8 \mathrm{~h}$ (e.g. Clarke et al. 2008; Radigan et al. 2012) which is consistent with an atmospheric feature rotating into and out of view. Other variables exhibit multi-component light curves (e.g. Artigau et al. 2009) that are suggestive of a rapid evolution of atmospheric features.

To investigate the variability of brown dwarfs across the full $\mathrm{L}-\mathrm{T}$ spectral sequence, we have performed a large-scale $J_{\mathrm{s}}$-band photometric monitoring campaign of 69 field brown dwarfs with the SofI instrument on the $3.5 \mathrm{~m}$ New Technology Telescope (NTT). This survey is a part of the BAM (Brown dwarf Atmosphere Monitoring) project. In Sect. 2, the properties of the sample, including magnitudes, spectral types, and companions are summarised. Details of the observations are reported in Sect. 3, followed by the data reduction procedure, and methodology used to characterise each target as variable or constant in Sect. 4. Section 5 presents the results of the program and a comparison to previous variability studies. Finally, we discuss the sensitivity of the BAM survey and investigate possible correlations between variability and various observables such as spectral type, colour and binarity in Sect. 6. The results are summarised in Sect. 7.

\section{The BAM sample}

The 69 objects in the BAM sample were drawn from the brown dwarf archive (dwarfarchives . org) and were selected to span the full sequence of L- and T-spectral types from L0 to T8. An equal proportion of targets with spectral types above, across and below the $\mathrm{L}-\mathrm{T}$ transition region were included. In this paper, we consider the $\mathrm{L}-\mathrm{T}$ transition to range from $\mathrm{L} 7$ to $\mathrm{T} 4$, following Golimowski et al. (2004). Spectral types including a fractional subtype have been rounded down - for example, an L6.5 is considered L6 for the statistics. For the 48 targets with parallax measurements (e.g. Dupuy \& Liu 2012; Faherty et al. 2012), a colour-magnitude diagram was constructed and is shown in Fig. 1. The histogram of target spectral types and a plot of the colour as a function of spectral type are shown in Fig. 2. The spectral types are based on IR spectroscopy for 54 targets and

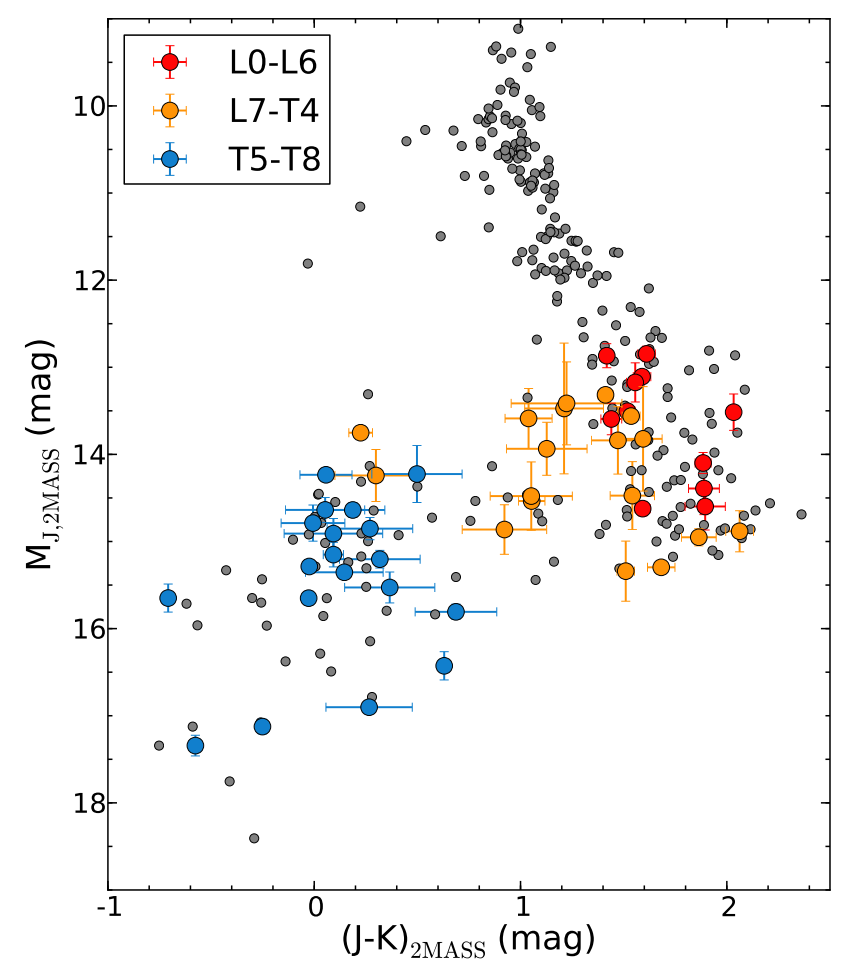

Fig. 1. Colour-magnitude diagram of the $\mathrm{M}-\mathrm{L}-\mathrm{T}$ spectrum (small grey circles). All brown dwarfs with known parallax in the BAM sample are overplotted, with red representing the L dwarfs, yellow the L-T transition dwarfs, and blue the T dwarfs (see Tables 1 and 2). Half spectral types have been rounded down in the study. The photometry and parallaxes for the field M-L-T objects are from Dupuy \& Liu (2012).

on optical spectroscopy for the remaining 15 targets that lacked an IR spectral classification. The spectral types, parallaxes, and apparent 2MASS magnitudes of the targets are listed in Table 1 for L dwarfs and Table 2 for T dwarfs.

Additional factors that influenced the target selection were the magnitudes and coordinates. To obtain high signal-to-noise individual measurements, the targets were limited to objects with magnitudes brighter than $J \sim 16.5 \mathrm{mag}$. To avoid observations at high airmass, the target declinations were limited to South of +20 degrees. Pairs of targets were observed for sequences of 2 to $4 \mathrm{~h}$, which also impacted the range of target coordinates observed each observing run.

The majority of the sample, 47 targets, have been observed in programs designed to detect binary companions with radial velocity variations (Blake et al. 2010), or spectra showing features of different spectral types (Burgasser et al. 2010), or high angular resolution imaging (i.e. Bouy et al. 2003; McCaughrean et al. 2004; Burgasser et al. 2003, 2005, 2006; Reid et al. 2006a, 2008; Looper et al. 2008). Based on the companion search programs reported in the literature, a total of 12 targets are members of spatially resolved binary pairs, and the results of all the binary searches are reported in Tables 1 and 2 . The proportion of binaries in this sample is comparable to the overall brown dwarf binary frequency (Burgasser et al. 2003), indicating that the sample is not biased in the level of multiple systems included. All of the binaries in the sample have separations less than the seeing limit, so the photometric measurements in this study record the combined flux from both components. A variable brown dwarf that is part of an unresolved binary system can be more difficult to detect, as the variability is diluted by the non variable companion. 

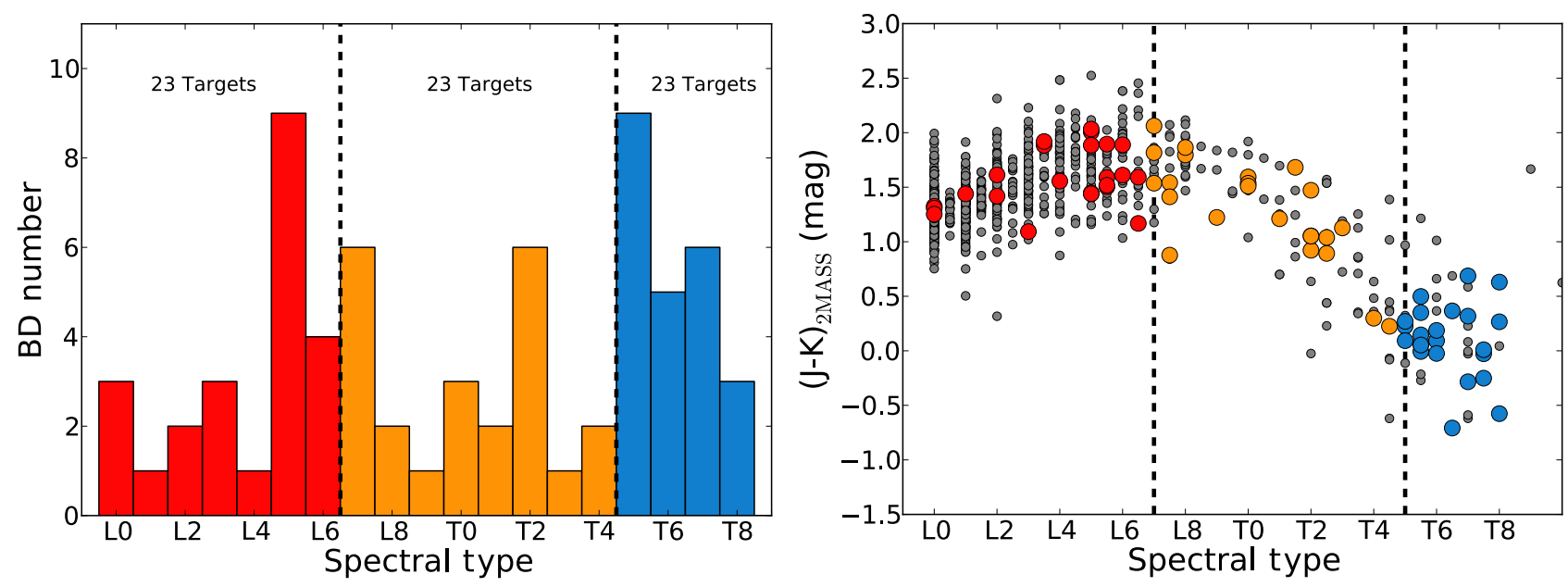

Fig. 2. Diagram on the left shows a histogram of the sample across their respective spectral classes, whilst on the right is a colour-colour diagram showing the $J-K$ colours of the same (coloured circles) overplotted on the full brown dwarf L-T spectral sequence (small grey circles). The L-T transition is indicated by the dashed lines defined in Golimowski et al. (2004).

Previous observations designed to search for photometric variability have been reported for approximately half the sample - 34 targets - and cover optical (Gelino et al. 2002; Koen 2013), near-IR (Enoch et al. 2003; Koen et al. 2004, 2005; Clarke et al. 2008; Khandrika et al. 2013; Buenzli et al. 2014), and radio (Berger 2006) wavelengths. It is important to note that the different variability monitoring studies apply different criteria to categorise a target as variable or constant, and a range of observation wavelengths have been employed. Most of the previous monitoring has been conducted over timescales of hours similar to this program, though a few studies covered longer timescales with lower cadence measurements (e.g. Gelino et al. 2002; Enoch et al. 2003).

\section{Observations}

The observations took place from 4 to 11 October 2011 and 3-9 April 2012 with the SofI (Son of ISAAC) instrument (Moorwood et al. 1998) mounted on the NTT (New Technology Telescope) at the ESO La Silla observatory. Observations were performed in the large field imaging mode that has a pixel scale of $0.288 \mathrm{px}^{-1}$ and a field-of-view of $4.92 \times 4.92$. During the first observing run, some of the targets were observed in both the $J_{\mathrm{s}}$-band and $K_{\mathrm{s}}$-band, but only the $J_{\mathrm{s}}$-band was used during the second run. As a consequence, six of the targets from the first run have $J_{\mathrm{s}}$-band data with lower cadence. The $J_{\mathrm{s}}$ filter $(1.16-1.32 \mu \mathrm{m})$ was used to avoid contamination by the water band centred at $1.4 \mu \mathrm{m}$ that would have otherwise affected the photometry. An increase in the telluric water column would have caused an anti-correlation between the brightness of the brown dwarfs and the reference stars in the $J$-band, since an increase in the water column will decrease the flux from the reference stars to a greater extent compared to the brown dwarfs that have deep intrinsic water bands. The $J_{\mathrm{s}}$ data should not suffer from this effect.

Three sets of two target fields were observed most nights, alternating between each target roughly every 15 min over a $\sim 3.5 \mathrm{~h}$ window. This procedure allowed six targets to be observed every night. During clear conditions, the observations had a detector integration time (DIT) of $5 \mathrm{~s}$, with three DITs (NDIT) taken and averaged together with about 25 exposures in each observing block. During poorer conditions, such as the presence of cirrus clouds, and for fainter objects, the exposure times were increased. The flux was kept below 10000 ADUs for the brightest targets in the field to prevent any non-linearity effects.

\section{Data reduction and photometry}

\subsection{Processing the images}

For each image, basic data reduction steps consisting of correcting for the dark current and division by a flat field and sky subtraction were applied. Developing flat field images for the NTT/SofI instrument involved generating two different flats, a special dome flat and an illumination correction flat as documented by the observatory. The dome flat requires observations of an evenly illuminated screen with the dome lamp turned on and off in a particular set sequence. To correct for low frequency sensitivity variations across the array that are not completely removed by the dome flat, an illumination correction was applied. By observing the flux from a standard star in a grid pattern across the array, a low order polynomial was fitted to the flux measurements, allowing large scale variations across the array to be characterised and removed. Flat field images were produced using the IRAF ${ }^{1}$ scripts provided by the observatory ${ }^{2}$. As the flat fields are documented to be extremely stable over several months, a single set of flat fields were used for all the targets in a given run.

For the SofI instrument, the dark frames are a poor estimate of the underlying bias pattern, which varies as a function of the incident flux. Consequently, the dark and bias are subtracted from the science frames through the computation of a sky frame, which also removes the sky background from the science data. Sky frames were generated by median combining the dithered science frames. The final calibration step involved measuring the offsets between the individual images and aligning all the science frames. The aligned frames within each $\sim 15 \mathrm{~min}$ interval were subsequently median combined. We compared the

\footnotetext{
1 IRAF is distributed by the National Optical Astronomy Observatories, which are operated by the Association of Universities for Research in Astronomy, Inc., under cooperative agreement with the National Science Foundation.

2 http://wWw. eso.org/sci/facilities/lasilla/ instruments/sofi/tools/reduction/sofi_scripts.html
} 
photometric uncertainties on the median combined images calculated using IRAF, to the standard deviation of the unbinned images within each bin. For most objects, the two methods for calculating uncertainties gave very similar results. The IRAF uncertainties on the median combined images were used for all the objects for consistency. Median combining the images before performing photometry rather than measuring the individual frames had the advantage of improving the centring, measurements of the full width at half maximum (FWHM), and the photometry of the fainter comparison stars in the field.

\subsection{Generating the light curves}

Aperture photometry was carried out using the APPHOT package in IRAF. A median value of the FWHM was measured per image using all the stars in the field-of-view. A range of aperture radii were explored and the size of $1.5 \times F W H M$ was selected, as it minimised the root-mean-square (rms) scatter of the reference star light curves that were created by dividing each reference star by the weighted mean of the remaining reference star light curves. The aperture was kept constant for all the stars in a single image, but was allowed to vary between individual images to account for variations in seeing. The variable aperture also yielded higher signal-to-noise measurements compared to a constant aperture, which would otherwise cause a loss in the flux measured within the aperture during poorer seeing conditions. We checked each target field to ensure that the photometry was not impacted by nearby astrophysical sources.

The steps taken to generate the target light curves in the survey are given in the following list:

- For each target, a list of reference star candidates was generated by considering all stars visible in the field of view, discarding stars with peak counts less than 20 ADUs or greater than 10000 ADUs. These limits were imposed to ensure enough signal was present to accurately centre the aperture around the object and to ensure that none of the reference stars were in the non-linear regime of the detector.

- Reference candidates were trimmed by selecting up to 15 of the reference stars with the most similar brightness to the target.

- Candidate reference star light curves were calculated by dividing each reference star by a weighted mean of the remaining reference stars.

- Candidate reference stars with light curves exhibiting a standard deviation greater or equal to the median standard deviation for all reference star candidates were removed.

- A master reference light curve was subsequently created by median combining the normalised light curves of all the qualifying reference stars.

- The final target light curve was produced by dividing the target brown dwarf flux by the weighted mean of all the qualifying reference stars. The light curve was normalised by dividing the light curve by the median flux value of the light curve.

- The target and reference star light curves were all airmass de-trended by dividing the light curves by a second order polynomial fit to the relative flux of the master reference as a function of airmass.

The number of reference stars used for each target is given in Tables 3 and 4, with six to eight references being typical. The automatic selection process was applied uniformly throughout the entire sample of objects. The uncertainties were calculated using IRAF. The target photometric uncertainty $(Q)$ is defined

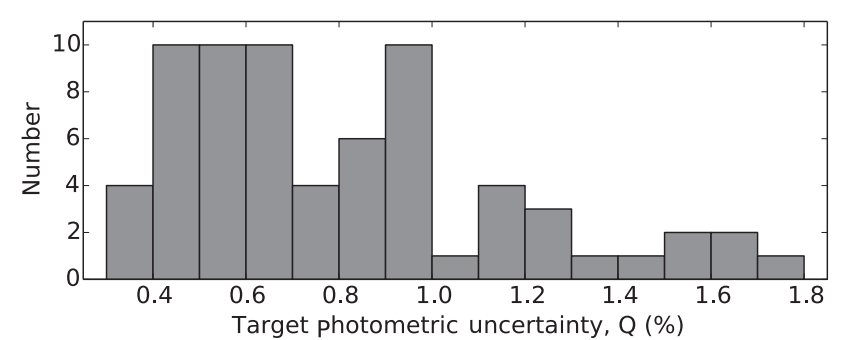

Fig. 3. Target photometric uncertainty of the survey defined as the median value of the final target light curve uncertainties. The median target photometric uncertainty is $0.7 \%$.

as the median value of the target light curve uncertainties. A histogram of the $Q$ values for each object is shown in Figure 3, and the value for all targets are listed in Table 3 and Table 4 . The median $Q$ value for the entire survey is $0.7 \%$.

\subsection{Identifying variables}

The significance of the variations were assessed in comparison to two criteria. For the first assessment, the final target light curve was compared against a flat line using the reduced robust median statistic ( $\tilde{\eta})$ (Enoch et al. 2003). The definition of $\tilde{\eta}$ is expressed as

$\tilde{\eta}=\frac{1}{d} \sum_{i=1}^{N}\left|\frac{\Delta F_{i}-\operatorname{median}(\Delta F)}{\sigma_{i}}\right|$

where $d$ defines the number of free parameters and $\sigma_{i}$, the uncertainty on each photometric measurement in the final target light curve.

For the second assessment, the reduced chi squared $\left(\chi_{v}^{2}\right)$ value for each target light curve was calculated relative to the master reference light curve. The definition of $\chi_{v}^{2}$ is expressed as

$\chi_{v}^{2}=\frac{1}{v} \sum_{i=1}^{N} \frac{\left(O_{i}-E_{i}\right)^{2}}{\sigma_{i}^{2}}$

where $v$ is the degrees of freedom, $O_{i}$ is the final target light curve, $E_{i}$ is the master reference light curve and $\sigma_{i}$ is the uncertainty on the final target light curve and master reference light curve added in quadrature.

Astrophysical variability was better determined calculating $\chi_{v}^{2}$ relative to the master reference light curve instead of a straight line, which was more prone to classifying variable conditions over intrinsic variability. We make use of the $\chi_{v}^{2}$ to estimate the cumulative distribution function and thus the $p$-value for each final target light curve. The $p$-value is the probability that the final target light curve is the same as ( $p$-value $>10 \%)$ or different from ( $p$-value $\leq 10 \%$ ) the master reference light curve. In Fig. 4, we plot the histogram of the calculated $p$-values for the full sample, and the large number of objects in the first bin gives an indication of the variables in the survey. The first bin contains 16 objects with $p$-value $\leq 5 \%$, and the level of false positives expected with an equivalent $p$-value is 3 to 4 objects $(5 \%)$ for a sample of 69 targets. For the identification of variables, both $p$-value and an $\tilde{\eta}$ threshold were applied. The number of targets with $p$-value $\leq 5 \%$ but $\tilde{\eta}>1$ was two, which is similar to the level of expected false positives. The excess of targets in the last two bins of Fig. 4 suggests that the uncertainties for the sample are conservatively estimated. 
Table 3. Variables identified in this study.

\begin{tabular}{|c|c|c|c|c|c|c|c|c|c|}
\hline Object & Spectral type & Obs. Dur. (h) & Refs. & DOF & $\chi_{v}^{2}$ & $\tilde{\eta}$ & $Q(\%)$ & $p$-value $(\%)$ & Amplitude $^{a}(\%)$ \\
\hline \multicolumn{10}{|c|}{ Variables with $p$-value $\leq 5 \%$ and $\tilde{\eta} \geq 1.0$} \\
\hline 2MASS J01062285-5933185 & L0 & 3.14 & 7 & 5 & 4.4 & 1.3 & 1.07 & 0.12 & $4.3 \pm 1.2$ \\
\hline 2MASS J13004255+1912354 & L3 & 3.06 & 5 & 9 & 3.6 & 2.6 & 1.21 & 0.0 & $9.6 \pm 0.9$ \\
\hline 2MASS J03582255-4116060 & L5 & 1.97 & 6 & 4 & 4.2 & 1.9 & 0.88 & 0.2 & $4.8 \pm 1.2$ \\
\hline 2MASS J08354256-0819237 & L5 & 3.32 & 6 & 10 & 5.8 & 2.0 & 0.33 & 0.0 & $1.7 \pm 0.5$ \\
\hline 2MASS J22551861-5713056 & L5.5 & 3.16 & 3 & 6 & 4.0 & 2.5 & 1.55 & 0.0 & $9.4 \pm 1.6$ \\
\hline 2MASS J10101480-0406499 & L6 & 3.08 & 4 & 8 & 4.3 & 2.0 & 1.18 & 0.0 & $5.1 \pm 1.1$ \\
\hline 2MASS J04390101-2353083 & L6.5 & 2.53 & 8 & 5 & 2.7 & 1.7 & 0.50 & 1.8 & $2.6 \pm 0.5$ \\
\hline 2MASS J11263991-5003550 & L6.5 & 3.23 & 8 & 12 & 3.3 & 1.4 & 0.52 & 0.0 & $3.2 \pm 0.7$ \\
\hline 2MASS J12074717+0244249 & T0 & 2.76 & 7 & 7 & 2.1 & 1.4 & 0.91 & 4.2 & $5.2 \pm 1.1$ \\
\hline 2MASS J21392676+0220226 & $\mathrm{T} 1.5$ & 2.62 & 7 & 7 & 18.1 & 3.9 & 0.42 & 0.0 & $4.7 \pm 0.5$ \\
\hline SIMP J013656.5+093347.3 & $\mathrm{T} 2.5$ & 2.94 & 5 & 7 & 2.3 & 2.2 & 0.72 & 2.3 & $3.0 \pm 0.6$ \\
\hline 2MASS J22282889-4310262 & T6.5 & 3.08 & 8 & 7 & 2.6 & 1.3 & 0.51 & 1.8 & $3.9 \pm 0.7$ \\
\hline 2MASS J00501994-3322402 & $\mathrm{T} 7.0$ & 2.63 & 6 & 6 & 5.6 & 3.3 & 1.24 & 0.0 & $10.8 \pm 1.3$ \\
\hline 2MASS J03480772-6022270 & $\mathrm{T} 7.0$ & 2.85 & 7 & 6 & 2.5 & 1.6 & 0.42 & 1.9 & $2.4 \pm 0.5$ \\
\hline \multicolumn{10}{|c|}{ Variables with $5 \%<p$-value $\leq 10 \%$ and $\tilde{\eta} \geq 1.0$} \\
\hline DENIS J0205.4-1159 & L5.5 & 3.22 & 5 & 5 & 2.0 & 1.5 & 0.50 & 7.9 & $2.0 \pm 0.6$ \\
\hline 2MASS J09310955+0327331 & L7.5 & 3.21 & 3 & 10 & 1.8 & 1.0 & 1.55 & 6.1 & $7.6 \pm 1.9$ \\
\hline 2MASS J12171110-0311131 & $\mathrm{T} 7.5$ & 2.78 & 6 & 5 & 1.9 & 1.2 & 0.93 & 9.3 & $4.2 \pm 1.1$ \\
\hline
\end{tabular}

Notes. ${ }^{(a)}$ These peak-to-trough amplitudes are calculated as the difference between the minimum and maximum points in the light curve. In some cases, these might represent the lower limit of the true amplitude, especially for brown dwarfs which exhibit variability on longer timescales.

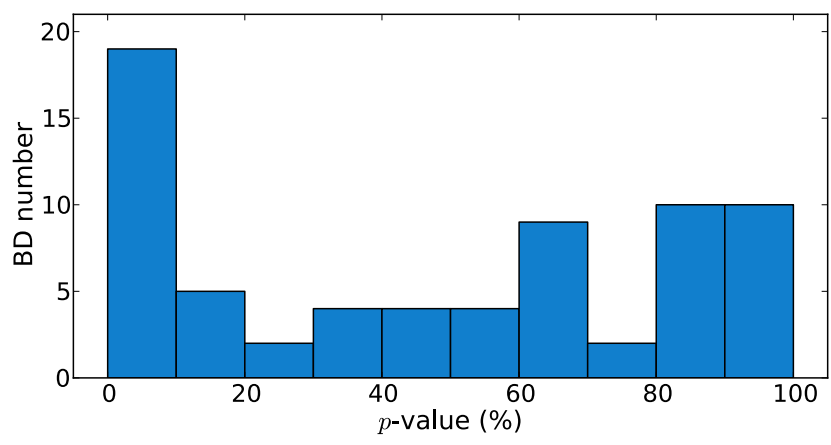

Fig. 4. $p$-value histogram of the full brown dwarf sample. The objects in the first bin include 16 targets with $p$-value $\leq 5 \%$, and three targets with $p$-value between 5-10\%. Of the 16 targets with $p$-value $\leq 5 \%$ listed in Table 3, two targets are not listed since they failed the robust criterion $(\tilde{\eta} \geq 1)$. The large number of objects in the last two bins (80 to 100) is suggestive of conservative error bars.

The $p$-value is the probability, under the assumption that we detect no variability (our null hypothesis), of observing variability greater or equal to what was observed in the master reference light curve. The survey has 39 targets satisfying the criterion of $\tilde{\eta} \geq 1$ and 16 targets with $p$-value $\leq 5 \%$. Objects classified as variable in this study satisfied two criteria, defined by $p$-value $\leq 5 \%$ and $\tilde{\eta} \geq 1$, and they are listed in Table 3 . Candidate variables with a less restrictive $p$-value $\leq 10 \%$ and $\tilde{\eta} \geq 1$ are also listed in Table 3 .

The min-to-max amplitudes of the variable objects were calculated as the difference between the highest and the lowest point in the light curve, using the uncertainties on these two points to calculate the uncertainty on the amplitude. Using this method to calculate the amplitude is dependent on how the data is binned, with the unbinned data showing larger amplitude variations. The amplitudes listed in Table 3 use the more conservative estimate from binned data. For objects with periods larger than the duration of observations, the amplitude is likely an underestimate, as the entire period is not observed. An example of a target with a known variable period exceeding the observation timescale is $2 \mathrm{M} 2139$, and the reported amplitude in this study is lower than longer timescale results (Radigan et al. 2012). Due to the limited duration and cadence of the observations, it is not possible to measure the periods of the variables in the BAM study.

\section{Results of the BAM survey}

The primary result from the BAM survey is the identification of a set of 14 variable brown dwarfs with $p$-value $\leq 5 \%$ and a further three candidate variables with $5 \%<p$-value $\leq 10 \%$ (see Sect. 5.1.1). For the remaining analysis, we only consider the $p$-value $\leq 5 \%$ variables. The BAM variables appear to show two morphologies. The first type of light curve shows pure sinusoidal trends, akin to $2 \mathrm{M} 2139$ (Radigan et al. 2012). Variables like 2M0050, 2M0348, 2M1010, and possibly 2M2255 appear to have sinusoidal light curves. The second group consists of targets that appear to be display multi-component variations in their light curves akin to SIMP0136 (Artigau et al. 2009; Metchev et al. 2013). SIMP0136 shows remarkable evolution in its features over multiple epochs, possibly caused by rapidly varying cloud features (Metchev et al. 2013). Objects with light curves similar to SIMP0136 object, such as 2M0106, 2M0439, 2M0835, 2M1126, 2M1207, 2M1300 are interesting for future follow up, to confirm whether or not they also show rapidly evolving light curves. Finally, the light curve of 2M0358 may be a fast rotating variable, since 2M0358 appears to oscillate through more than one cycle within the limited timespan of the BAM monitoring. The light curve of 2M2228 shows similar short time scale variations as $2 \mathrm{M} 0358$ and was previously found to be variable with a period of $P=1.43_{-0.15}^{+0.16} \mathrm{~h}$ by Clarke et al. (2008). Final target light curves for the 14 BAM variables and the associated comparison master reference light curves are shown in Fig. 5. Similar plots for the candidate variables are given in Fig. 6. The amplitudes and $p$-values are reported in Table 3.

Representative light curves of nine constant targets with a range of photometric qualities are shown in Fig. 7. These light 

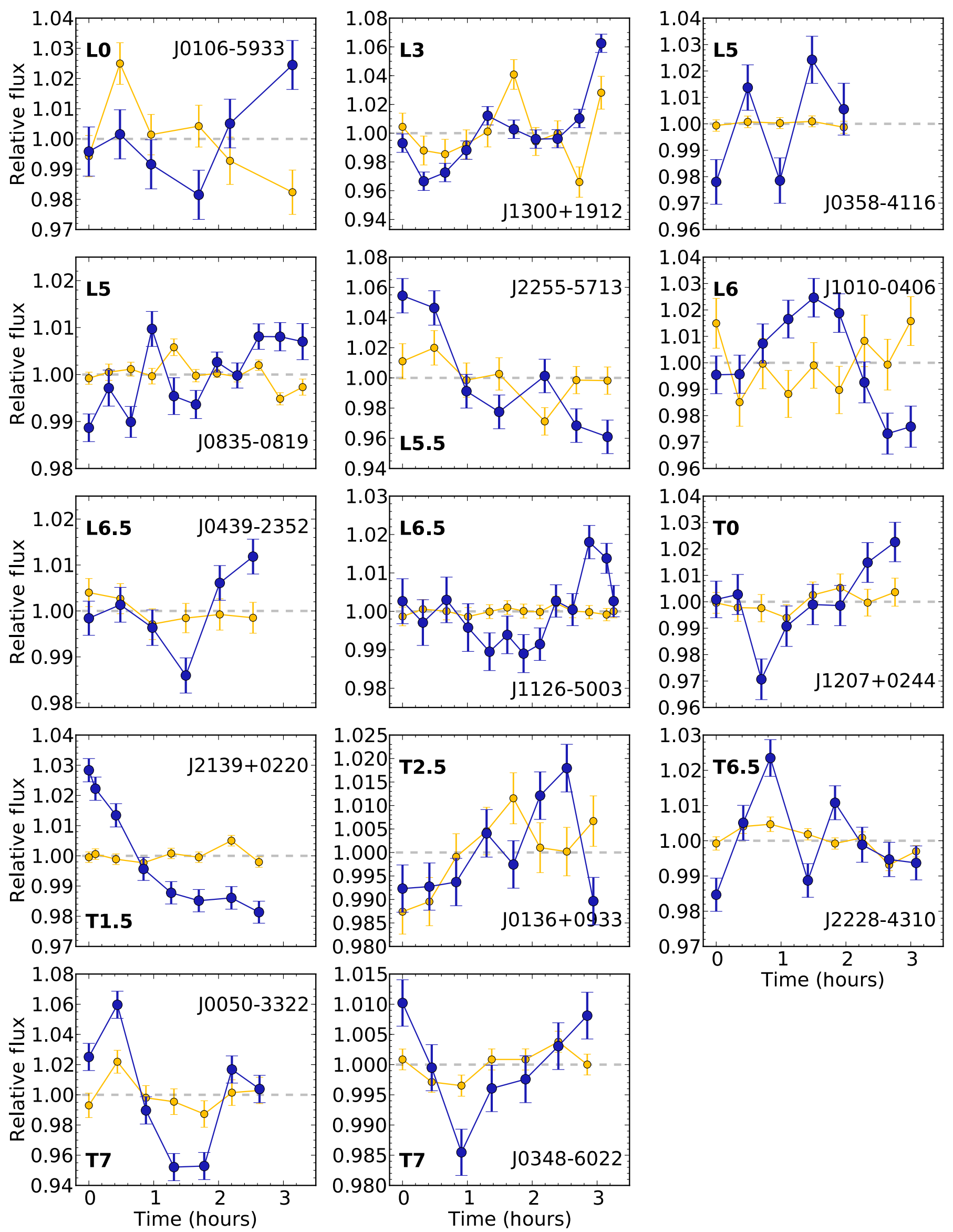

Fig. 5. Final target light curves of the 14 variable objects (blue points) with a $p$-value $\leq 5 \%$ and $\tilde{\eta} \geq 1.0$ together with the master reference light curves (yellow points). The uncertainties on the variable light curve incorporates the uncertainties in the master reference light curve. 
P. A. Wilson et al.: The brown dwarf atmosphere monitoring (BAM) project. I.
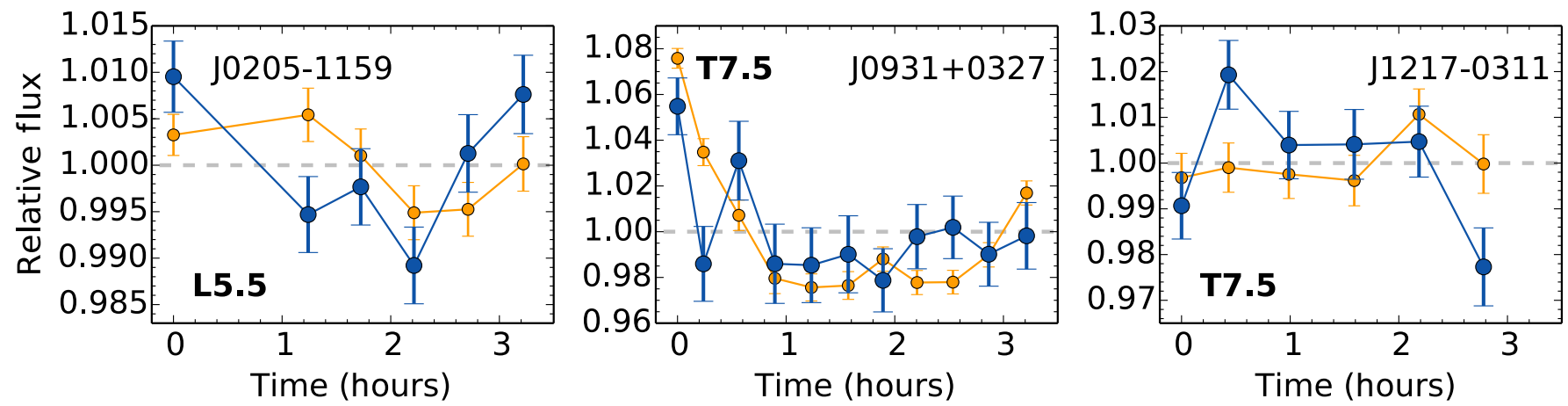

Fig. 6. Final target light curves of the candidate variables (larger blue points) with $5 \%<p$-value $\leq 10 \%$ and $\tilde{\eta} \geq 1.0$ together with the master reference light curves (yellow smaller points).
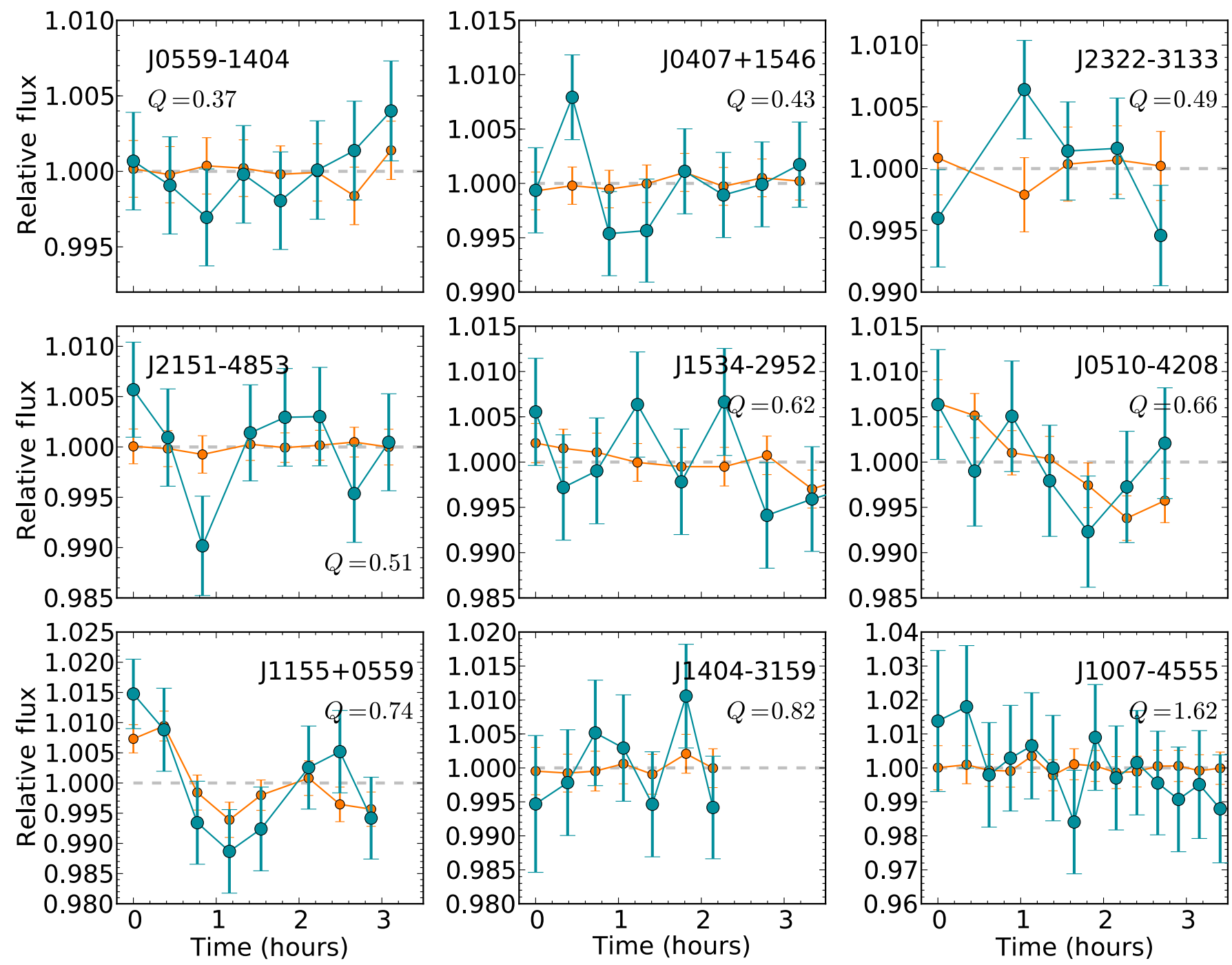

Fig. 7. Final target light curves of a subset of constant objects in this survey (larger blue points) with the master reference light curves (yellow smaller points). The target photometric uncertainty decreases from top to bottom and includes the light curve with the best photometry (top left) and light curve with the worst photometry (bottom right).

curves show the full range of the data quality for brown dwarfs of similar brightness to the variables identified in the study. The constant light curves are not all flat, however their variations are not statistically distinct from their associated master reference. The constant targets do not satisfy the two separate criteria used to identify the variables (specified in Sect. 4.3) which require the final target light curve to be distinct from the master reference light curve ( $p$-value) and a flat line $(\tilde{\eta})$. The $p$-values for constant sources are given in Table 4.

Since the $p$-value $\leq 5 \%$ cutoff is a statistical measure, there remains a likelihood of a contamination level of 3-4 false variables that are statistical fluctuations, $\sim 5 \%$ of the entire sample. Continued monitoring of the variables should help identify false positives. 


\subsection{Comparison of variables with previous studies}

This $J_{\mathrm{s}}$-band SofI program is the largest uniform monitoring survey conducted in the near-IR. Several previous surveys have targeted smaller sample sets (e.g. Enoch et al. 2003; Koen et al. 2004, 2005; Clarke et al. 2008; Khandrika et al. 2013; Girardin et al. 2013; Buenzli et al. 2014) or searched in different wavelengths such as I-band (e.g. Gelino et al. 2002; Koen 2004, 2013). Apart from results of the study by Koen (2013), previous surveys have typically targeted fewer than $\sim 25$ objects and detected variability frequencies of $\sim 30 \%$ in their sample sets, with a significant amount of overlap in the target samples used in different studies (Khandrika et al. 2013). The BAM sample was designed to uniformly cover the $\mathrm{L}-\mathrm{T}$ spectral range (see Fig. 2) and includes 35 brown dwarfs that have not been previously monitored in different surveys. There are nine new BAM variables, six of which have not been previously monitored for variability - 2M0050, 2M0106, 2M0358, 2M1010, 2M1207 and $2 \mathrm{M} 2255$. The survey has three variables that were previously found to be constant, and found nine brown dwarfs previously classified as variable to be constant. Finally, there are five variables that were found to vary in the literature and in this BAM study. A synopsis of the variables in the BAM and previous surveys is presented in Table 5. These objects are used to investigate the persistence of variability in Sect. 6.5. Table 6 presents the constant brown dwarf sample in the BAM study. These are targets that were monitored in previous surveys and were found to be constant in the literature and in this study. In the following two subsections, we compare our results with literature measurements for the variables identified in this sample and in previous work.

\subsubsection{BAM variables}

In Table 5, we present information for all the targets that were considered variable, either in this BAM study or in the literature. Three of the BAM variables - 2M0348, 2M0439 and 2M1126 were identified as constant brown dwarfs in prior surveys but appear to be variable in this survey. A further five brown dwarfs - SIMP0136, 2M0835, 2M1300, 2M2139 and 2M2228 - were confirmed to be variable both in this study and in the literature. Of these five, SIMP0136, 2M2139 and 2M2228 were previously found to vary in the near-IR, similar to this study. The other two variables 2M0835 and 2M1300 were originally measured to vary in the $I_{\mathrm{c}}$ band, and also display multi-component variations at near-IR wavelengths. Amongst the known variables with measured periods (from previous studies), only 2M2139 and 2M1300 have periods longer than the duration of the BAM monitoring data $(>7 \mathrm{~h}$, and $238 \mathrm{~h}$, respectively). The latter period is much longer than the expected rotation for a brown dwarf (Zapatero Osorio et al. 2006), which indicates that the periodic feature might not be related to the rotation of the brown dwarf (Gelino et al. 2002). The three remaining targets with previously measured periods - SIMP0136, 2M0835 and 2M2228 - were monitored in this study with a time span greater than one of their rotational periods. All three objects that we monitored over an entire period had amplitudes consistent with what has been previously published.

\subsubsection{Variables in previous studies not confirmed with BAM}

There are nine targets from the BAM sample that have been previously reported as variable, but were found to be constant in this survey. These sources are listed in Table 5, with a summary of the previous results pertaining to variability, including the observation wavelength and any notes on the amplitudes and timescales of the variations in brightness. One of these nine variables from literature - 2M0228 - has only been monitored in the optical. The remaining eight variables exhibited modulations in the near-IR. 2M0559, 2M0624, DENIS0817 and 2M1624 were found to have small amplitude variations in the Buenzli et al. (2014) survey carried out using the HST grism data. In the HST survey, 2M1624 showed variability in the water band $(1.35-1.44 \mu \mathrm{m})$ but was found to be constant at $J$-band wavelengths. Similar to other ground based surveys that found some of these targets constant, this BAM survey likely does not have the photometric sensitivity necessary to confirm the HST variables, nor is it possible to monitor the water bands from ground. Another four targets - SDSS0423, 2M0939, 2M1534 and 2M2331 - also appear constant in the data. The photometric uncertainties on SDSS0423 and 2M2331 are too large to confirm their lower amplitude variability of $\sim 0.8 \%$ and $\sim 1.2 \%$, respectively. Despite 2M0939 having been observed as a variable in the $K^{\prime}$ band with an amplitude of $3.1 \%$ (Khandrika et al. 2013), we are unable to confirm any variability in $J_{\mathrm{s}}$ with the BAM observations. 2M1534 was detected to vary in the $J H K_{\mathrm{s}}$ bands initially in (Koen et al. 2004), but was constant in a later epoch (Koen et al. 2005). Koen (2013) further discounts the likelihood of detecting short period variability in $2 \mathrm{M} 1534$, but maintains that the target likely varies on the timescale of a few days. The reported amplitudes in the $H$-band and $K$-band are below the detection threshold in the data for this target.

\section{Discussion}

\subsection{The sensitivity of the BAM survey}

To obtain an estimate of the variability frequency for brown dwarfs across spectral types, it is essential to quantify the sensitivity of the data to detecting different amplitudes of variability. We estimate the sensitivity to variables of a certain amplitude as three times the target photometric uncertainty of each final target light curve. This places a limit on the minimum amplitude required for a detection above a certain statistical significance threshold. The proportion of the sample that is sensitive to a given variability amplitude is shown as a function of amplitude in Fig. 8. As shown in Fig. 8, the BAM survey is capable of detecting any object in the sample showing a peak-to-trough amplitude $\geq 2.3 \%$ during the duration of the observations. The detection probability continues to decrease with decreasing amplitude with a sensitivity of $50 \%$ occurring for variables with a $\sim 1.7 \%$ amplitude. Given that the full BAM sample is sensitive to variables with amplitudes $\geq 2.3 \%$, Table 7 quantifies the frequency of variability for different subsets of spectral types using an amplitude cutoff of $2.3 \%$ and $p$-value $\leq 0.05$; this level includes all but one BAM $p \leq 0.05$ variable. Figure 9 shows how the variability frequency (considering all spectral types) varies as a function of amplitude to account for the declining proportion of the sample that is sensitive to lower amplitude variables.

To calculate the uncertainty on the variability frequency we use the binomial distribution

$B\left(n ; N, \epsilon_{v}\right)=\frac{N !}{n !(N-n) !} \epsilon_{v}^{n}\left(1-\epsilon_{v}\right)^{N-n}$.

where $n$ is the number of variables, $N$ the sample size and $\epsilon_{v}$ the variability frequency. This approach is based on Bayes' theorem under the assumption of a uniform prior based on no a priori knowledge and is ideal for small samples such as is the case for the BAM survey. 
P. A. Wilson et al.: The brown dwarf atmosphere monitoring (BAM) project. I.

Table 5. Summary of variable sources.

\begin{tabular}{|c|c|c|c|c|}
\hline Target name & Band & Variable/constant & References & Notes \\
\hline \multicolumn{5}{|c|}{ New variables from this study with no prior observations } \\
\hline 2MASS J00501994-3322402 & $J_{\mathrm{S}}$ & $\mathrm{V}$ & & \multirow{7}{*}{ Candidate variable $(p-v a l=6.1 \%)$} \\
\hline 2MASS J01062285-5933185 & $J_{\mathrm{s}}$ & $\mathrm{V}$ & & \\
\hline 2MASS J03582255-4116060 & $J_{\mathrm{s}}$ & V & & \\
\hline 2MASS J09310955+0327331 & $J_{\mathrm{s}}$ & $\mathrm{V}$ & & \\
\hline 2MASS J10101480-0406499 & $J_{\mathrm{s}}$ & $\mathrm{V}$ & & \\
\hline 2MASS J12074717+0244249 & $J_{\mathrm{s}}$ & V & & \\
\hline 2MASS J22551861-5713056 & $J_{\mathrm{s}}$ & $\mathrm{V}$ & & \\
\hline \multicolumn{5}{|c|}{ New variables previously categorised as constant } \\
\hline DENIS J0205.4-1159 & $I_{\mathrm{c}}$ & $\mathrm{C}$ & K13 & \\
\hline \multirow[t]{2}{*}{ Candidate Variable $(p$-val $=7.9 \%)$} & $K_{\mathrm{s}}$ & $\mathrm{C}$ & E03 & $<3 \%$ \\
\hline & $8.46 \mathrm{GHz}$ & $\mathrm{C}$ & B06 & $<30 \mu \mathrm{Jy}$ \\
\hline 2MASS J03480772-6022270 & $J$ & $\mathrm{C}$ & $\mathrm{C} 08$ & $<10$ mmag, periodic \\
\hline \multirow{3}{*}{ 2MASS J04390101-2353083 } & $I_{\mathrm{c}}$ & $\mathrm{C}$ & K13 & \\
\hline & $I_{\mathrm{c}}$ & $\mathrm{C}$ & K05 & \\
\hline & $8.46 \mathrm{GHz}$ & $\mathrm{C}$ & B06 & $<42 \mu \mathrm{Jy}$ \\
\hline 2MASS J11263991-5003550 & $I_{\mathrm{c}}$ & $\mathrm{C}$ & K13 & possibly periodic \\
\hline \multicolumn{5}{|c|}{ Literature variables confirmed as variable in this study } \\
\hline SIMP J013656.5+093347.3 & $J K$ & $\mathrm{~V}$ & A09, Ap13 & $\Delta J=4.5 \%, P=2.39 \pm 0.05 \mathrm{hr}$ \\
\hline \multirow{2}{*}{ 2MASS J08354256-0819237 } & $I_{\mathrm{c}}$ & $\mathrm{V}$ & K04, K13 & $\Delta I_{\mathrm{c}}=10-16 \mathrm{mmag}, P=3.1 \mathrm{hr}$ \\
\hline & $8.46 \mathrm{GHz}$ & $\mathrm{C}$ & B06 & $<30 \mu \mathrm{Jy}$ \\
\hline 2MASS J12171110-0311131 & $J$ & $\mathrm{~V}$ & A03 & $\Delta J=0.176 \pm 0.013 \mathrm{mag}$ \\
\hline \multirow[t]{2}{*}{ Candidate Variable $(\mathrm{p}-\mathrm{val}=9.3 \%)$} & $8.46 \mathrm{GHz}$ & $\mathrm{C}$ & B06 & $<111 \mu \mathrm{Jy}$ \\
\hline & & & Z06 & \\
\hline \multirow{6}{*}{ 2MASS J13004255+1912354 } & $I_{\mathrm{c}}$ & $\mathrm{C}$ & K13 & \\
\hline & $I$ & $\mathrm{~V}$ & G02 & $P=238 \pm 9 \mathrm{hr}$ \\
\hline & $8.46 \mathrm{GHz}$ & $\mathrm{C}$ & B06 & $<87 \mu \mathrm{Jy}$ \\
\hline & $J+K^{\prime}$ & $\mathrm{C}$ & Kh13 & $J<1.1 \%, K^{\prime}<1.7 \%$ \\
\hline & $I_{\mathrm{c}}$ & $\mathrm{C}$ & K05 & \\
\hline & $J H K_{\mathrm{s}}$ & $\mathrm{C}$ & KMM04 & \\
\hline \multirow{2}{*}{ 2MASS J21392676+0220226 } & $J H K_{\mathrm{s}}$ & $\mathrm{V}$ & R12, Ap13 & $\Delta(J, H, K)=(0.3,0.18,0.17) \mathrm{mag}, P=7.721 \pm 0.005 \mathrm{hr}$ \\
\hline & $J+K$ & $\mathrm{~V}$ & Kh13 & $\Delta J=6.7 \%, \mathrm{C}$ at $K^{\prime}<8 \%$ \\
\hline \multirow[t]{2}{*}{ 2MASS J22282889-4310262 } & $J$ & $\mathrm{~V}$ & $\mathrm{C} 08, \mathrm{Bu} 12$ & $\Delta J=15.4 \pm 1.4 \mathrm{mmag}, P=1.43 \pm 0.16 \mathrm{hr}$ \\
\hline & $8.46 \mathrm{GHz}$ & $\mathrm{C}$ & B06 & $<30 \mu \mathrm{Jy}$ \\
\hline \multicolumn{5}{|c|}{ Objects with reported IR variability measured as constants in this study } \\
\hline \multirow[t]{5}{*}{ SDSS J042348.56-041403.5 } & $I_{\mathrm{c}}$ & $\mathrm{C}$ & K13 & \\
\hline & $K_{\mathrm{s}}$ & likely V & E03 & $0.3 \pm 0.18 \mathrm{mag}, P=1.39-1.62 \mathrm{hr}$ \\
\hline & $J$ & $\mathrm{~V}$ & $\mathrm{C} 08$ & $8.0 \pm 0.8 \mathrm{mmag}, \mathrm{P}=2 \pm 0.4 \mathrm{hr}$ \\
\hline & $J H K_{\mathrm{s}}$ & $\mathrm{C}$ & KTTK05 & $J<15 \mathrm{mmag}, H<11 \mathrm{mmag}, K<2 \mathrm{mmag}$ \\
\hline & $8.46 \mathrm{GHz}$ & $\mathrm{C}$ & B06 & $<42 \mu \mathrm{Jy}$ \\
\hline \multirow[t]{6}{*}{ 2MASS J05591914-1404488 } & HST G141 grism & $\mathrm{V}$ & Bu13 & \\
\hline & $I_{\mathrm{c}}$ & $\mathrm{C}$ & K13 & \\
\hline & $K_{\mathrm{s}}$ & $\mathrm{C}$ & E03 & $<7 \%$ \\
\hline & $J$ & $\mathrm{C} 08$ & $\mathrm{C}$ & $<5 \mathrm{mmag}$ \\
\hline & $I_{\mathrm{c}}$ & $\mathrm{C}$ & K04 & \\
\hline & $8.46 \mathrm{GHz}$ & B06 & $<27 \mu \mathrm{Jy}$ & \\
\hline 2MASS J06244595-4521548 & HST G141 grism & $\mathrm{V}$ & $\mathrm{Bu} 13$ & \\
\hline DENIS J081730.0-615520 & HST G141 grism & $\mathrm{V}$ & Bu13 & \\
\hline \multirow[t]{2}{*}{ 2MASS J09393548-2448279 } & $K^{\prime}$ & $\mathrm{V}$ & Kh13 & $0.31 \mathrm{mag}$ \\
\hline & $J$ & $\mathrm{C}$ & & $<0.141 \mathrm{mag}$ \\
\hline \multirow[t]{5}{*}{ 2MASS J15344984-2952274 } & $I_{\mathrm{c}}$ & $\mathrm{C}$ & K13 & \\
\hline & $I_{\mathrm{c}}$ & $\mathrm{C}$ & K05 & \\
\hline & $J H K_{\mathrm{s}}$ & $\mathrm{C}$ & KTTK05 & $J<10 \mathrm{mmag}, H<11 \mathrm{mmag}, K<18 \mathrm{mmag}$ \\
\hline & $J H K_{\mathrm{s}}$ & $\mathrm{V}$ & KMM04 & $H 4 \mathrm{mmag}, K 7 \mathrm{mmag}, P=0.96 \mathrm{hr}$ \\
\hline & $8.46 \mathrm{GHz}$ & $\mathrm{C}$ & B06 & $<63 \mu \mathrm{Jy}$ \\
\hline 2MASS J16241436+0029158 & HST G141 grism & $\mathrm{V}$ & Bu13 & Variability detected in water band $(1.35-1.44 \mu \mathrm{m})$ \\
\hline & $J H K_{\mathrm{s}}$ & $\mathrm{C}$ & KMM04 & \\
\hline & $8.46 \mathrm{GHz}$ & $\mathrm{C}$ & B06 & $<36 \mu \mathrm{Jy}$ \\
\hline 2MASS J23312378-4718274 & $J$ & $\mathrm{~V}$ & $\mathrm{C} 08$ & $\Delta J=12.4 \pm 1.3 \mathrm{mmag}, P=2.9 \pm 0.9 \mathrm{hr}$ \\
\hline & Objects with repo & ed optical variabilit & measured as c & nstants in this study \\
\hline 2MASS J02284355-6325052 & $I_{\mathrm{c}}$ & $\mathrm{V}$ & K13 & \\
\hline
\end{tabular}

References. Artigau et al. (2003) [A03]; Artigau et al. (2009) [A09]; Apai et al. (2013) [Ap13]; Berger (2006) [B06]; Buenzli et al. (2012) [Bu12]; Buenzli et al. (2014) [Bu13]; Clarke et al. (2008) [C08]; Enoch et al. (2003) [E03]; Gelino et al. (2002) [G02]; Khandrika et al. (2013) [Kh13]; Koen (2004) [K04]; Koen et al. (2004) [KMM04]; Koen et al. (2005) [KTTK05]; Koen (2005) [K05]; Koen (2013) [K13]; Radigan et al. (2012) [R12]; Zapatero Osorio et al. (2006) [Z06].

The rotation period is another factor that can influence the detectability of a variable signal. In Fig. 10, we present the results of simulating light curves to test the detection probability of the survey to brown dwarf variables with different periods. 
Table 6. Summary of constant sources.

\begin{tabular}{|c|c|c|c|}
\hline Target name & Band & References & Notes \\
\hline 2MASS J02572581-3105523 & $I_{\mathrm{c}}$ & K13 & \\
\hline 2MASS J04070752+1546457 & $J K^{\prime}$ & Kh13 & no results \\
\hline 2MASS J04151954-0935066 & $8.46 \mathrm{GHz}$ & B06 & $<\mu \mathrm{Jy}$ \\
\hline \multirow[t]{3}{*}{ 2MASS J04455387-3048204 } & $I_{\mathrm{c}}$ & K13 & \\
\hline & $I_{\mathrm{c}}$ & K04 & \\
\hline & $8.46 \mathrm{GHz}$ & B06 & $<66 \mu \mathrm{Jy}$ \\
\hline \multirow[t]{3}{*}{ 2MASS J05233822-1403022 } & $I_{\mathrm{c}}$ & K13 & \\
\hline & $I_{\mathrm{c}}$ & K05 & \\
\hline & $8.46 \mathrm{GHz}$ & B06 & $231 \pm 14 \mu \mathrm{Jy}$ \\
\hline \multirow[t]{4}{*}{ 2MASS J12255432-2739466 } & $J$ & KTTK05 & $<12 \mathrm{mmag}$ \\
\hline & $H$ & & $<14 \mathrm{mmag}$ \\
\hline & $K_{\mathrm{s}}$ & & $<9 \mathrm{mmag}$ \\
\hline & $J H K_{\mathrm{s}}$ & KMM04 & \\
\hline \multirow[t]{2}{*}{ 2MASS J12281523-1547342 } & $I_{\mathrm{c}}$ & K13 & \\
\hline & $8.46 \mathrm{GHz}$ & B06 & $<87 \mu \mathrm{Jy}$ \\
\hline \multirow[t]{2}{*}{ 2MASS J12545393-0122474 } & $J H K_{\mathrm{s}}$ & KMM04 & \\
\hline & $J$ & Gi13 & $<5 \mathrm{mmag}$ \\
\hline \multirow[t]{3}{*}{ 2MASS J15074769-1627386 } & $I_{\mathrm{c}}$ & K13 & \\
\hline & $I_{\mathrm{c}}$ & K03 & \\
\hline & $8.46 \mathrm{GHz}$ & B06 & $<57 \mu \mathrm{Jy}$ \\
\hline \multirow[t]{2}{*}{ CFBDS J151114+060742 } & $J$ & Kh13 & $<3.3 \%$ \\
\hline & $K^{\prime}$ & & $<6.1 \%$ \\
\hline 2MASS J15462718-3325111 & $J H K_{\mathrm{s}}$ & KMM04 & \\
\hline 2MASS J15530228+1532369 & $J H K_{\mathrm{s}}$ & KMM04 & \\
\hline \multirow[t]{2}{*}{ 2MASS J16322911+1904407 } & $8.46 \mathrm{GHz}$ & B06 & $<54 \mu \mathrm{Jy}$ \\
\hline & HST G141 Grism & Bu13 & \\
\hline 2MASS J19360187-5502322 & $I_{\mathrm{c}}$ & K13 & \\
\hline 2MASS J23224684-3133231 & $I_{\mathrm{c}}$ & K13 & \\
\hline
\end{tabular}

References. Berger (2006) [B06]; Buenzli et al. (2014) [Bu13]; Girardin et al. (2013) [Gi13]; Khandrika et al. (2013) [Kh13]; Koen (2004) [K04]; Koen (2003) [K03]; Koen et al. (2004) [KMM04]; Koen et al. (2005) [KTTK05]; Koen (2005) [K05]; Koen (2013) [K13].

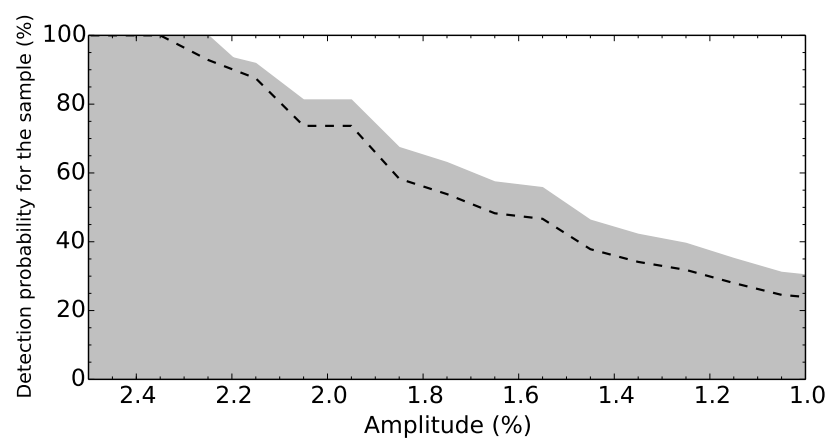

Fig. 8. Proportion of the survey sensitive to variability as a function of peak-to-trough amplitudes for different detection thresholds. The dashed line represents the fraction of objects with a photometric accuracy good enough to have allowed for the detection of variability. The shaded area represents the region of sensitivity with the upper binomial errors and amplitude uncertainties added to the variability fraction.

We simulated sinusoidal light curves with three different amplitudes, of $1.5 \%, 2.5 \%$, and $5.0 \%$, and with periods ranging from a minimum of 1 hour to a maximum of $12 \mathrm{~h}$ (Zapatero Osorio et al. 2006). Gaussian noise equal to the median photometric uncertainty of the survey of $0.7 \%$ was added to each light curve. To mimic the binned SofI data, the light curves were sampled at intervals of 15 minutes, and each simulated dataset was divided into groups of $3 \mathrm{~h}$, similar to the typical duration of the BAM data. For light curves with period longer than $3 \mathrm{~h}$, we generated multiple datasets, by stepping through the sine curve in steps of 5 degrees of phase and calculating the $p$-value at each phase, ensuring full sampling of the phase. Figure 10 shows the

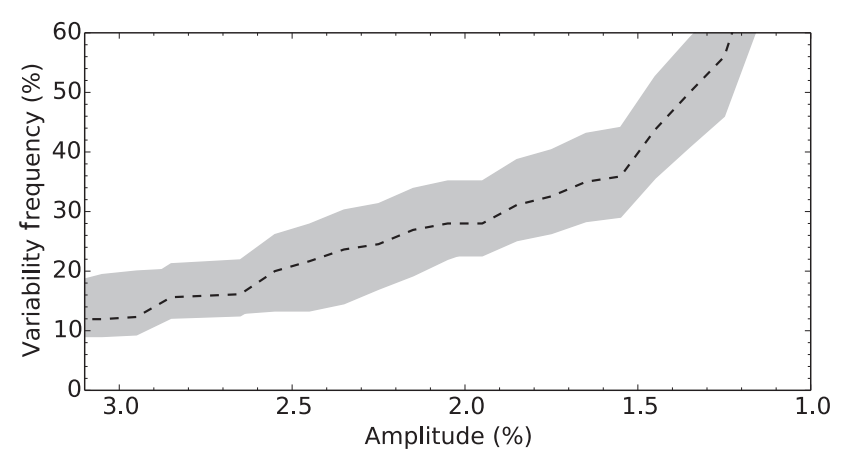

Fig. 9. Variability frequency as a function of amplitude (dashed line) with the binomial errors and amplitude uncertainties added to the variability fraction (shaded area).

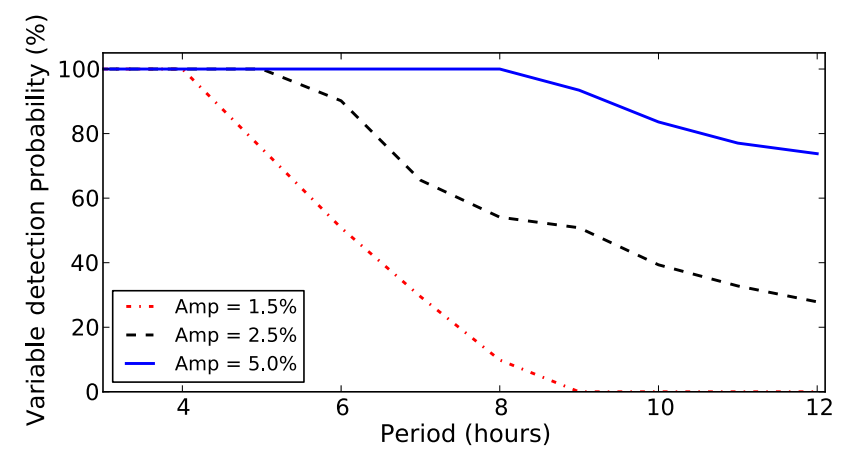

Fig. 10. Percentage of simulated sinusoidal light curves detected as variable, as a function of period from $1 \mathrm{~h}$ to $12 \mathrm{~h}$, for three different amplitudes. We used the measured survey median noise of $0.7 \%$, and each sine curve was sampled at intervals of $15 \mathrm{~min}$ to imitate the binned data of the survey. Additionally, we stepped through each sine curve at 5 degree phase intervals, to ensure that we sampled the full phase of the variable light curve.

percentage of simulated light curves that are detected as variable with a $p$-value $\leq 5 \%$. For amplitudes of $5.0 \%$, periodicities from 3 to $12 \mathrm{~h}$ are easily recovered with a probability of 80 to $100 \%$, while the required periods decrease to $\sim 6$ hours for $2.5 \%$ variables and $\sim 5 \mathrm{~h}$ for $1.5 \%$ variables for detection probabilities in the 80 to $100 \%$ range.

\subsection{Frequency and amplitude of variability across spectral types}

The frequency of variables as a function of spectral type is an important topic, since models of brown dwarf atmospheres have suggested that breakup of clouds across the L-T transition may result in both a higher rate of occurrence and a higher amplitude of variability compared to earlier $\mathrm{L}$ and later $\mathrm{T}$ objects. Amongst the previously known variables, the two largest amplitude variable objects discovered to-date are $\mathrm{L}-\mathrm{T}$ transition objects - SIMP0136 ( 5\% in $J$-band but with a significant night to night evolution, Artigau et al. 2009) and 2M2139 (as high as $26 \%$ in the $J$-band, Radigan et al. 2012).

As indicated by the variability frequencies reported in Table 7, the BAM results show no evidence that the frequency of variables in the L7 to T4 transition region is distinct from the earlier spectral types, the later spectral types, or the combination of all non-transition region brown dwarfs. The variability frequencies in Table 7 are calculated using the entire sample of targets and an amplitude threshold of $\geq 2.3 \%$ and $p \leq 0.05$. 
Table 7. Variability frequency.

\begin{tabular}{lcccc}
\hline \hline Sample & Sp. type & No. targets & No. variables $^{a}$ & Freq. (\%) $^{+11}$ \\
\hline Early-L & L0-L6 & 23 & 7 & $30_{-8}^{+10}$ \\
Late-T & T5-T8 & 23 & 3 & $13_{-4}^{+10}$ \\
L-T Transition & L7-T4 & 23 & 3 & $13_{-4}^{+10}$ \\
Outside L-T transition & L0-L6 \& T5-T8 & 46 & 10 & $22_{-5}^{+7}$ \\
\hline
\end{tabular}

Notes. ${ }^{(a)}$ These are the variables with a $p$-value $\leq 5 \%$, and amplitude $\geq 2.3 \%$.
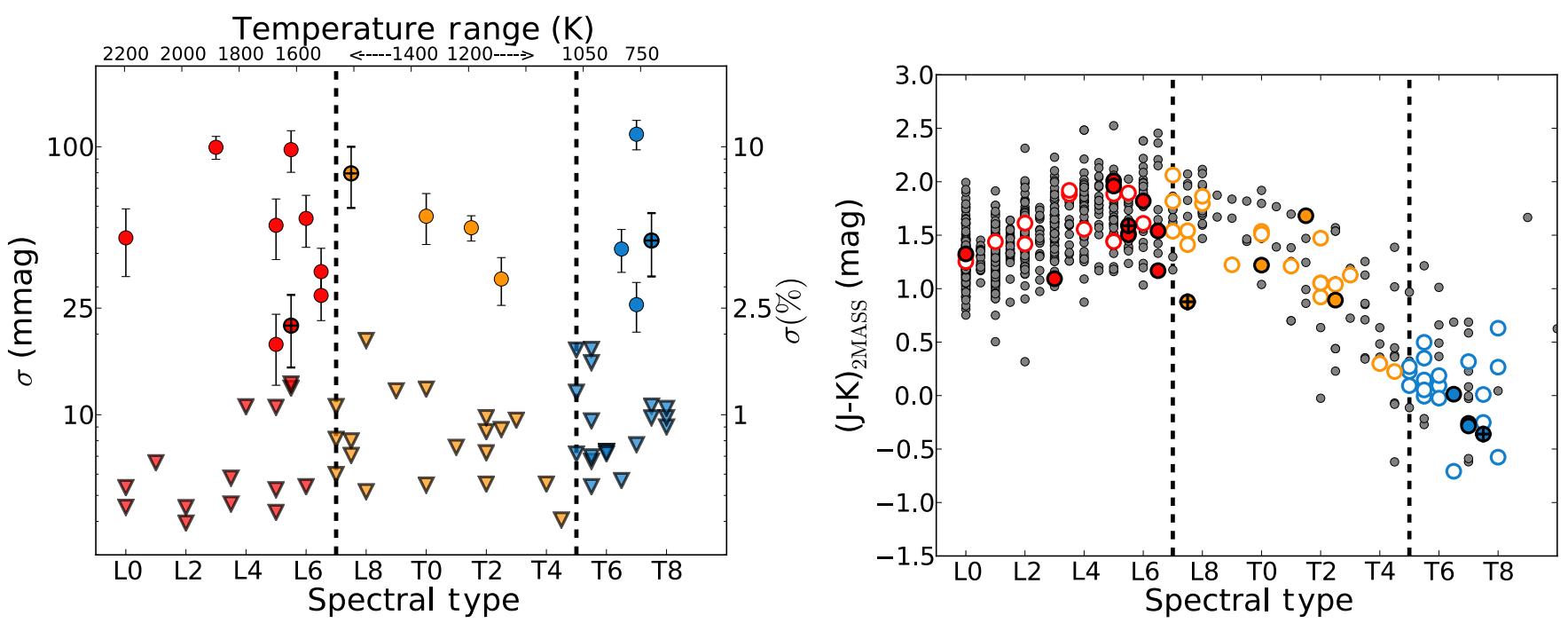

Fig. 11. Left: amplitude of the variables ( $p$-value $\leq 5 \%$ as closed circles and $5 \%<p$-value $\leq 10 \%$ as closed circles with cross) and target photometric uncertainty of the non-varying objects (coloured triangles) across the entire spectral range of the sample. Right: colour-colour diagram of the entire $\mathrm{L}$ through $\mathrm{T}$ spectral range with the full sample plotted with open circles, showing the colour spread of the targets. The variables from the BAM sample are overplotted ( $p$-value $\leq 5 \%$ as closed circles and $5 \%<p$-value $\leq 10 \%$ as closed circles with cross). The L-T transition is indicated by the dashed lines.

Although no statistically significant difference in the variability frequencies for transition brown dwarfs is measured with the BAM survey, the $2.3 \%$ amplitude limit of the analysis would not have detected differences at lower amplitudes, and removing the peak-to-trough amplitude threshold does not change this result. Adjusting the boundaries of the transition region by up to two spectral types does not change the result. Likewise, the amplitudes of the detected variables show no clear trends with spectral type within the capacity of the survey, as shown in Fig. 11 (left).

The BAM variability frequency is very comparable to estimates for M stars. A variability frequency between $\sim 21-29 \%$ for $19 \mathrm{M}$-stars was measured in a multi-wavelgenth optical study with the Calar Alto Observatory in Spain (Rockenfeller et al. 2006). The wavelength of observations for the M-star study was shorter than the BAM survey $J$-band data, and the amplitude of variations is expected to decline for longer wavelengths (e.g. Reiners et al. 2010).

In a recent compilation of variability surveys, Khandrika et al. (2013) reported a variability frequency of $30 \pm 5 \%$ based on a collection of different surveys with observations obtained in the optical and near-IR passbands, covering 78 objects in total. Comparison between surveys is difficult as the variability frequency may depend on a variety of different factors, including the target selection criterion and the criteria used to define variability in the targets which usually differs from one survey to the next. Additionally, the observed wavelength may also alter the variability frequency with different wavelength probing different depths in the atmosphere. Koen (2013) finds a poor overlap between the variables identified with optical and near-IR filters (of the 13 variables already observed in near-IR surveys, 7 were found as constant and 6 as variable in the optical). Because of the uniform sensitivity of this survey, we did not incorporate the results of previous studies into the statistics, presented in Table 7. The presence of highly variable objects outside the transition region, may suggest the possibility of both early onset of cloud condensation in the atmospheres of mid-L dwarfs and the emergence of sulfide clouds in mid-T dwarfs (Morley et al. 2012). Other physical processes that have been suggested to possibly induce variability in the atmospheres of brown dwarfs include coupling clouds with global atmosphere circulation (Showman \& Kaspi 2013; Zhang \& Showman 2014), and variability caused by thermal perturbations emitted from deeper layers within the brown dwarf atmosphere (Robinson \& Marley 2014).

\subsection{Variability as a function of colour within a spectral type}

The $J-K$ colour of the sample as a function of the spectral type is shown in Figure 11 (right). The targets span nearly the full colour spread in early-L, transition and late-T sub sample. The 14 BAM variables and the three candidates are not clustered toward either the red or the blue within any particular spectral type. Previous studies (e.g. Khandrika et al. 2013) have suggested that brown dwarfs with unusual colours (highly red or blue) compared to the median of the spectral type might be indicative of 
variable cloud cover. We performed a two sample K-S test to determine whether or not the detrended colours of the BAM variables were distinct from the rest of the sample. The maximum difference between the cumulative distributions was 0.18 with a corresponding $p$-value of $\sim 75 \%$, indicating that the two datasets are consistent with being drawn from the same sample. The BAM study thus finds no correlation between the variables and the colour of a brown dwarf within each spectral type.

\subsection{Binarity and variability}

The BAM sample includes 12 confirmed binaries out of 47 targets studied for binarity with another four SpeX spectra binary candidates. Including the binary candidates, 10 out of the 16 binaries in the BAM sample fall in the L-T transition. This is consistent with previous detections of an increase in the binary frequency across the L-T transition Burgasser et al. (2006). Amongst the BAM variables, only 2M2255 is a confirmed binary, while 2M1207 and 2M2139 are binary candidates. Five of the variables are confirmed to be single, and six have not been studied for binarity. The limited data provides no evidence to support a correlation between variability and binarity amongst the objects in the BAM survey.

\subsection{Persistence of variability}

A recent multi-epoch ( $\sim 4$ years) monitoring study of the variable brown dwarf SIMP0136 (Metchev et al. 2013) revealed that the target has significant evolution in its light curve, changing from highly variable to constant in a 2 month period. When compared to the SofI light curve for SIMP0136, the target shows a fascinating variation in amplitude. It appears to be variable at $3 \%$ in the SofI data, while a month later it shows large amplitude variations $(\sim 9 \%)$ in the $J$-band, only to appear constant a few months later. Similar night-to-night variations have also been seen in SDSS J105213.51+442255.7 (Girardin et al. 2013). The evolution indicates a lack of persistence in the source of variability over timescales longer than a few weeks and it suggests that the brown dwarfs identified as constant in this study might similarly exhibit periods of quiescence and enhanced activity. The BAM survey only examines variability on the timescale of a single rotation period or less as compared to some surveys (e.g. Gelino et al. 2002; Enoch et al. 2003) that study the flux variations of brown dwarfs over longer timescales.

The BAM data, in combination with previous results, can be used to address the question of persistence of variability. Table 8 summarises the observations related to persistence of variability, using information presented in Tables 5 and 6. For greatest consistency with the BAM study, we consider other epochs of near-IR data rather than optical. A total of of 34 BAM targets have an earlier epoch of observation. 2M0228 is the only source measured to be variable in the optical $\left(I_{\mathrm{c}}\right)$ that switched from variable to constant. Table 8 indicates that brown dwarf variability does not necessarily persist on longer timescales, with only half the BAM variables showing variation in both epochs. The survey finds four previously constant objects to be variable and nine targets previously reported as variable in the literature to be constant, making these ideal candidates for multiple epoch monitoring programs.
Table 8. Summary of persistence results.

\begin{tabular}{lc}
\hline \hline Total targets with 2 epochs & 34 \\
\hline Variable at 2 epochs & 6 \\
Constant at 2 epochs & 15 \\
Switch between variable and constant & 13 \\
\hline
\end{tabular}

\section{Summary}

We present the results of the largest near-IR brown dwarf variability survey conducted in the $J_{\mathrm{s}}$-band using the NTT $3.5 \mathrm{~m}$ telescope. The BAM survey has an unbiased sample of 69 early$\mathrm{L}$ through late-T brown dwarfs. A total of 14 variable objects were detected: six new variables not previously studied for variability, three objects previously reported as constant, and five previously known variables. The nine newly identified variables constitute a significant increase in the total number of known brown dwarf variables characterisable using ground-based facilities. In a recent study of 57 L4-T9 brown dwarfs (Radigan et al. 2014), a set of 35 targets were observed by both studies, enabling a direct comparison of results. Of the 35 targets in both samples, both studies classify a common 26 targets as not variable and a common 4 targets as variable. Of the 5 remaining variables noted in a single study ( 2 in BAM, 3 in Radigan et al. 2014), 4 can be explained by differences in sensitivity for the specific light curves.

Rather than quoting a single number for the variability frequency, we discuss how the frequency of variable brown dwarfs depends on different factors such as the observed wavelength and the variability amplitude. The BAM study, representing the largest and most uniform ground-based search for variability, was designed to address the important question of the physical properties of brown dwarf atmospheres including the L-T transition. One class of models has suggested that this colour change, that defines the transition, may be a manifestation of the breakup of clouds resulting in a patchy coverage across the surface (Ackerman \& Marley 2001), which would have the observable consequence of enhanced variability at the $\mathrm{L}-\mathrm{T}$ transition. Considering the results of this study, covering both transition and non-transition objects and statistical significance of the variability, there is no distinction between the variability frequency between the brown dwarfs in the transition region or outside the transition region. This suggests that the patchy cloud scenario may not provide the full explanation for the $\mathrm{L}-\mathrm{T}$ transition or that the induced level of variability is substantially below the detection thresholds of the current study. The 14 variables, including the nine newly identified variables, will provide valuable systems with which to pursue additional questions of the physics of brown dwarf atmospheres, including the longitudinal and vertical variations of clouds and active regions which can be inferred from multi-wavelength follow-up monitoring.

Acknowledgements. Based on observations made with ESO Telescopes at the La Silla Paranal Observatory under programme ID 188.C-0493. We would like to thank the anonymous referee for valuable suggestions that helped improve this paper. PAW acknowledges support from STFC. J.P. was supported by a Leverhulme research project grant (F/00144/BJ), and funding from an STFC standard grant. This research has made use of the SIMBAD database and VizieR catalogue access tool, CDS, Strasbourg, France. The original description of the VizieR service was published in Ochsenbein et al. 2000, A\&AS 143, 23. This research has benefitted from the $\mathrm{M}, \mathrm{L}, \mathrm{T}$, and $\mathrm{Y}$ dwarf compendium housed at DwarfArchives.org. This research made use of Astropy, a community-developed core Python package for Astronomy (Robitaille et al. 2013). We thank F. Pont, R. De Rosa and D. K. Sing for valuable feedback and discussion. 
P. A. Wilson et al.: The brown dwarf atmosphere monitoring (BAM) project. I.

\section{References}

Ackerman, A. S., \& Marley, M. S. 2001, ApJ, 556, 872

Albert, L., Artigau, É., Delorme, P., et al. 2011, AJ, 141, 203

Andrei, A. H., Smart, R. L., Penna, J. L., et al. 2011, AJ, 141, 54

Apai, D., Radigan, J., Buenzli, E., et al. 2013, ApJ, 768, 121

Artigau, É., Nadeau, D., \& Doyon, R. 2003, in Brown Dwarfs, ed. E. Martín, IAU Symp., 211, 451

Artigau, É., Doyon, R., Lafrenière, D., et al. 2006, ApJ, 651, L57

Artigau, É., Bouchard, S., Doyon, R., \& Lafrenière, D. 2009, ApJ, 701, 1534

Artigau, É., Radigan, J., Folkes, S., et al. 2010, ApJ, 718, L38

Bailer-Jones, C. A. L. 2005, in 13th Cambridge Workshop on Cool Stars, Stellar Systems and the Sun, eds. F. Favata, G. A. J. Hussain, \& B. Battrick, ESA SP, 560,429

Baraffe, I., Chabrier, G., Barman, T. S., Allard, F., \& Hauschildt, P. H. 2003, A\&A, 402, 701

Barman, T. S., Macintosh, B., Konopacky, Q. M., \& Marois, C. 2011, ApJ, 733, 65

Berger, E. 2006, ApJ, 648, 629

Blake, C. H., Charbonneau, D., \& White, R. J. 2010, ApJ, 723, 684

Bouy, H., Brandner, W., Martín, E. L., et al. 2003, AJ, 126, 1526

Buenzli, E., Apai, D., Morley, C. V., et al. 2012, ApJ, 760, L31

Buenzli, E., Apai, D., Radigan, J., Reid, I. N., \& Flateau, D. 2014, ApJ, 782, 77

Burgasser, A. J., Kirkpatrick, J. D., Brown, M. E., et al. 1999, ApJ, 522, L65

Burgasser, A. J., Wilson, J. C., Kirkpatrick, J. D., et al. 2000, AJ, 120, 1100

Burgasser, A. J., Kirkpatrick, J. D., Reid, I. N., et al. 2003, ApJ, 586, 512

Burgasser, A. J., McElwain, M. W., Kirkpatrick, J. D., et al. 2004, AJ, 127, 2856

Burgasser, A. J., Reid, I. N., Leggett, S. K., et al. 2005, ApJ, 634, L177

Burgasser, A. J., Kirkpatrick, J. D., Cruz, K. L., et al. 2006, ApJS, 166, 585

Burgasser, A. J., Looper, D. L., Kirkpatrick, J. D., Cruz, K. L., \& Swift, B. J. 2008, ApJ, 674, 451

Burgasser, A. J., Cruz, K. L., Cushing, M., et al. 2010, ApJ, 710, 1142

Burningham, B., Pinfield, D. J., Lucas, P. W., et al. 2010, MNRAS, 406, 1885

Burrows, A., Sudarsky, D., \& Hubeny, I. 2006, ApJ, 640, 1063

Chabrier, G., \& Baraffe, I. 2000, ARA\&A, 38, 337

Chiu, K., Fan, X., Leggett, S. K., et al. 2006, AJ, 131, 2722

Clarke, F. J., Hodgkin, S. T., Oppenheimer, B. R., Robertson, J., \& Haubois, X. 2008, MNRAS, 386, 2009

Cruz, K. L., Reid, I. N., Liebert, J., Kirkpatrick, J. D., \& Lowrance, P. J. 2003, AJ, 126, 2421

Cruz, K. L., Reid, I. N., Kirkpatrick, J. D., et al. 2007, AJ, 133, 439

Cushing, M. C., Kirkpatrick, J. D., Gelino, C. R., et al. 2011, ApJ, 743, 50

Dahn, C. C., Harris, H. C., Vrba, F. J., et al. 2002, AJ, 124, 1170

Deacon, N. R., \& Hambly, N. C. 2007, A\&A, 468, 163

Delfosse, X., Tinney, C. G., Forveille, T., et al. 1997, A\&A, 327, L25

Dupuy, T. J., \& Liu, M. C. 2012, ApJS, 201, 19

Ellis, S. C., Tinney, C. G., Burgasser, A. J., Kirkpatrick, J. D., \& McElwain, M. W. 2005, AJ, 130, 2347

Enoch, M. L., Brown, M. E., \& Burgasser, A. J. 2003, AJ, 126, 1006

Faherty, J. K., Burgasser, A. J., Walter, F. M., et al. 2012, ApJ, 752, 56

Fan, X., Knapp, G. R., Strauss, M. A., et al. 2000, AJ, 119, 928

Folkes, S. L., Pinfield, D. J., Kendall, T. R., \& Jones, H. R. A. 2007, MNRAS, 378,901

Gelino, C. R., Marley, M. S., Holtzman, J. A., Ackerman, A. S., \& Lodders, K. 2002, ApJ, 577, 433

Girardin, F., Artigau, É., \& Doyon, R. 2013, ApJ, 767, 61

Gizis, J. E. 2002, ApJ, 575, 484
Gizis, J. E., Monet, D. G., Reid, I. N., et al. 2000, AJ, 120, 1085

Golimowski, D. A., Leggett, S. K., Marley, M. S., et al. 2004, AJ, 127, 3516

Hawley, S. L., Covey, K. R., Knapp, G. R., et al. 2002, AJ, 123, 3409

Kendall, T. R., Delfosse, X., Martín, E. L., \& Forveille, T. 2004, A\&A, 416, L17

Kendall, T. R., Jones, H. R. A., Pinfield, D. J., et al. 2007, MNRAS, 374, 445

Khandrika, H., Burgasser, A. J., Melis, C., et al. 2013, AJ, 145, 71

Kirkpatrick, J. D., Reid, I. N., Liebert, J., et al. 1999, ApJ, 519, 802

Kirkpatrick, J. D., Cruz, K. L., Barman, T. S., et al. 2008, ApJ, 689, 1295

Knapp, G. R., Leggett, S. K., Fan, X., et al. 2004, AJ, 127, 3553

Koen, C. 2003, MNRAS, 346, 473

Koen, C. 2004, MNRAS, 354, 378

Koen, C. 2005, MNRAS, 360, 1132

Koen, C. 2013, MNRAS, 428, 2824

Koen, C., Matsunaga, N., \& Menzies, J. 2004, MNRAS, 354, 466

Koen, C., Tanabé, T., Tamura, M., \& Kusakabe, N. 2005, MNRAS, 362, 727

Leggett, S. K., Geballe, T. R., Fan, X., et al. 2000, ApJ, 536, L35

Looper, D. L., Kirkpatrick, J. D., \& Burgasser, A. J. 2007, AJ, 134, 1162

Looper, D. L., Gelino, C. R., Burgasser, A. J., \& Kirkpatrick, J. D. 2008, ApJ, 685, 1183

Marley, M. S., Seager, S., Saumon, D., et al. 2002, ApJ, 568, 335

McCaughrean, M. J., Close, L. M., Scholz, R.-D., et al. 2004, A\&A, 413, 1029

Metchev, S., Apai, D., Radigan, J., et al. 2013, Astron. Nachr., 334, 40

Moorwood, A., Cuby, J.-G., \& Lidman, C. 1998, The Messenger, 91, 9

Morales-Calderón, M., Stauffer, J. R., Kirkpatrick, J. D., et al. 2006, ApJ, 653, 1454

Morley, C. V., Fortney, J. J., Marley, M. S., et al. 2012, ApJ, 756, 172

Radigan, J., Jayawardhana, R., Lafrenière, D., et al. 2012, ApJ, 750, 105

Radigan, J., Lafrenière, D., Jayawardhana, R., \& Artigau, E. 2014, ApJ, submitted [arXiv: 1404.3247]

Reid, I. N., Kirkpatrick, J. D., Gizis, J. E., et al. 2000, AJ, 119, 369

Reid, I. N., Lewitus, E., Allen, P. R., Cruz, K. L., \& Burgasser, A. J. 2006a, AJ, 132,891

Reid, I. N., Lewitus, E., Burgasser, A. J., \& Cruz, K. L. 2006b, ApJ, 639, 1114

Reid, I. N., Cruz, K. L., Burgasser, A. J., \& Liu, M. C. 2008, AJ, 135, 580

Reiners, A., Bean, J. L., Huber, K. F., et al. 2010, ApJ, 710, 432

Robinson, T. D., \& Marley, M. S. 2014, ApJ, 785, 158

Robitaille, T. P., Tollerud, E. J., Astropy Collaboration, et al. 2013, A\&A, 558, A33

Rockenfeller, B., Bailer-Jones, C. A. L., \& Mundt, R. 2006, A\&A, 448, 1111

Scholz, R.-D., McCaughrean, M. J., Lodieu, N., \& Kuhlbrodt, B. 2003, A\&A, 398, L29

Showman, A. P., \& Kaspi, Y. 2013, ApJ, 776, 85

Sing, D. K., Désert, J.-M., Lecavelier Des Etangs, A., et al. 2009, A\&A, 505, 891

Sing, D. K., Pont, F., Aigrain, S., et al. 2011, MNRAS, 416, 1443

Smart, R. L., Tinney, C. G., Bucciarelli, B., et al. 2013, MNRAS, 433, 2054

Stephens, D. C., \& Leggett, S. K. 2004, PASP, 116, 9

Strauss, M. A., Fan, X., Gunn, J. E., et al. 1999, ApJ, 522, L61

Stumpf, M. B., Geißler, K., Bouy, H., et al. 2011, A\&A, 525, A123

Tinney, C. G., \& Tolley, A. J. 1999, MNRAS, 304, 119

Tinney, C. G., Burgasser, A. J., \& Kirkpatrick, J. D. 2003, AJ, 126, 975

Tinney, C. G., Burgasser, A. J., Kirkpatrick, J. D., \& McElwain, M. W. 2005, AJ, 130, 2326

Vrba, F. J., Henden, A. A., Luginbuhl, C. B., et al. 2004, AJ, 127, 2948

Zapatero Osorio, M. R., Martín, E. L., Bouy, H., et al. 2006, ApJ, 647, 1405

Zhang, X., \& Showman, A. P. 2014 [arXiv: 1403.2143]

Pages 14 to 16 are available in the electronic edition of the journal at http://www . aanda.org 
Table 1. L dwarf sample.

\begin{tabular}{|c|c|c|c|c|c|c|}
\hline Target name & $\begin{array}{l}\text { Spectral } \\
\text { type }\end{array}$ & $\begin{array}{c}\text { Parallax } \\
\text { (mas) }\end{array}$ & $\begin{array}{r}J_{\text {2MASS }} \\
(\mathrm{mag})\end{array}$ & $\begin{array}{c}\text { Binary/ } \\
\text { Single }\end{array}$ & Instrument & References \\
\hline 2MASS J00165953-4056541 & $\mathrm{L} 3.5^{a}$ & & $15.316 \pm 0.061$ & $\mathrm{~S}$ & SpeX spectra & $\mathrm{K} 08, \mathrm{BC} 10$ \\
\hline 2MASS J00184613-6356122 & $\mathrm{L} 2^{a}$ & & $15.224 \pm 0.044$ & & & R08 \\
\hline 2MASS J01062285-5933185 & $\mathrm{L}^{a}{ }^{a}$ & & $14.33 \pm 0.035$ & & & R08 \\
\hline 2MASS J01282664-5545343 & L1 & $15.24 \pm 7.9$ & $13.775 \pm 0.027$ & & & K07, D07 \\
\hline DENIS J0205.4-1159 & L5.5 & $50.6 \pm 1.5$ & $14.587 \pm 0.03$ & B (409 mas) & HST imaging & R06, K04, D02, B03 \\
\hline 2MASS J02284355-6325052 & Lo & & $13.556 \pm 0.028$ & & & K07 \\
\hline 2MASS J02572581-3105523 & $\mathrm{L}^{a}{ }^{a}$ & & $14.672 \pm 0.039$ & & & K08, R08 \\
\hline 2MASS J03185403-3421292 & $\mathrm{L}^{a}{ }^{a}$ & $72.9 \pm 7.7$ & $15.569 \pm 0.055$ & S & HST imaging & K08, F12, R08 \\
\hline 2MASS J03400942-6724051 & $\mathrm{L}^{a}{ }^{a}$ & & $14.742 \pm 0.032$ & $\mathrm{~S}$ & SpeX spectra & $\begin{array}{c}\text { BC10 } \\
\text { C07, K08 }\end{array}$ \\
\hline 2MASS J03582255-4116060 & $\mathrm{L}^{a}$ & & $15.846 \pm 0.087$ & & & R08 \\
\hline 2MASS J04070752+1546457 & L3.5 & & $15.478 \pm 0.058$ & & & R08, B06 \\
\hline 2MASS J04390101-2353083 & $\mathrm{L}_{6} .5^{a}$ & $110.4 \pm 4.0$ & $14.408 \pm 0.029$ & $\mathrm{~S}$ & HST imaging & $\mathrm{C} 03, \mathrm{~F} 12, \mathrm{R} 06$ \\
\hline 2MASS J04455387-3048204 & $\mathrm{L} 2^{a}$ & $78.5 \pm 4.9$ & $13.393 \pm 0.026$ & $\mathrm{~S}$ & HST imaging & $\mathrm{C} 03, \mathrm{~F} 12, \mathrm{R} 06$ \\
\hline 2MASS J05233822-1403022 & L5 & & $13.084 \pm 0.024$ & $\mathrm{~S}$ & radial velocity & C03, W03, BCW10 \\
\hline 2MASS J06244595-4521548 & $\mathrm{L}^{a}{ }^{a}$ & $83.9 \pm 4.5$ & $14.48 \pm 0.029$ & $\mathrm{~S}$ & HST imaging & R08, F12, R06 \\
\hline 2MASS J08354256-0819237 & $\mathrm{L}^{a}$ & $117.3 \pm 11.2$ & $13.169 \pm 0.024$ & $\begin{array}{l}S \\
S\end{array}$ & $\begin{array}{l}\text { HST imaging } \\
\text { radial velocity }\end{array}$ & $\begin{array}{c}\text { C03, And11, R06 } \\
\text { BCW10 }\end{array}$ \\
\hline & & & & S & SpeX spectra & $\mathrm{BC} 10$ \\
\hline 2MASS J09153413+0422045 & $\mathrm{L} 7^{a}$ & & $14.548 \pm 0.03$ & B (730 mas) & HST imaging & R08, R06 \\
\hline 2MASS J09310955+0327331 & L7.5 & & $16.615 \pm 0.138$ & & & K04 \\
\hline 2MASS J10043929-3335189 & $\mathrm{L} 4^{a}$ & $54.8 \pm 5.6$ & $14.48 \pm 0.035$ & & & Giz02, And11 \\
\hline 2MASS J10101480-0406499 & $\mathrm{L}^{a}{ }^{a}$ & $59.8 \pm 8.1$ & $15.508 \pm 0.059$ & & & $\mathrm{C} 03, \mathrm{~F} 12$ \\
\hline 2MASS J11263991-5003550 & L6.5 & & $13.997 \pm 0.032$ & & & F07, B06 \\
\hline 2MASS J11555389+0559577 & L7.5 & $57.9 \pm 10.2$ & $15.66 \pm 0.077$ & $\mathrm{~S}$ & SpeX spectra & $\mathrm{K} 04, \mathrm{~F} 12, \mathrm{BC} 10$ \\
\hline 2MASS J12281523-1547342 & L6 & $44.8 \pm 1.8$ & $14.378 \pm 0.03$ & B (264 mas) & HST imaging & D97, K04, D12, B03 \\
\hline 2MASS J13004255+1912354 & L3 & & $12.717 \pm 0.022$ & $\begin{array}{l}\mathrm{S} \\
\mathrm{S}\end{array}$ & $\begin{array}{l}\text { HST imaging } \\
\text { radial velocity }\end{array}$ & $\begin{array}{c}\text { Giz00, B06, R08 } \\
\text { BCW10 }\end{array}$ \\
\hline 2MASS J13262981-0038314 & L5.5 & $50 \pm 6$ & $16.103 \pm 0.071$ & $\mathrm{~S}$ & SpeX spectra & $\mathrm{F} 00, \mathrm{~K} 04, \mathrm{~V} 04, \mathrm{BC} 10$ \\
\hline 2MASS J15074769-1627386 & L5.5 & $136.4 \pm 0.6$ & $12.83 \pm 0.027$ & $\mathrm{~S}$ & HST imaging & R00, K04, D02, R06 \\
\hline 2MASS J16322911+1904407 & L8 & $65.6 \pm 2.1$ & $15.867 \pm 0.07$ & $\mathrm{~S}$ & HST imaging & K99, B06, D02, B03 \\
\hline 2MASS J19360187-5502322 & $\mathrm{L}^{a}{ }^{a}$ & $66.3 \pm 5.4$ & $14.486 \pm 0.039$ & $\mathrm{~S}$ & HST imaging & R08, F12, R06 \\
\hline SDSS J204317.69-155103.4 & L9 & $22.8 \pm 4.7$ & $16.625 \pm 0.162$ & $\mathrm{~S}$ & SpeX spectra & $\mathrm{C} 06, \mathrm{~S} 13, \mathrm{BC} 10$ \\
\hline 2MASS J22521073-1730134 & L7.5 & $63.2 \pm 1.6$ & $14.313 \pm 0.029$ & B (130 mas) & HST imaging & Ken04, D12, RL06 \\
\hline 2MASS J22551861-5713056 & L5.5 & & $14.083 \pm 0.03$ & $\mathrm{~B}(120 \mathrm{mas})$ & HST imaging & K07, R08 \\
\hline 2MASS J23224684-3133231 & $\mathrm{L}^{a}{ }^{a}$ & $58.6 \pm 5.6$ & $13.577 \pm 0.027$ & & & $\mathrm{R} 08, \mathrm{~F} 12$ \\
\hline
\end{tabular}

Notes. ${ }^{(a)}$ Spectral classification using optical data.

References. Andrei et al. (2011) [And11]; Bouy et al. (2003) [B03]; Blake et al. (2010) [BCW10]; Berger (2006) [B06]; Burgasser et al. (2010) [BC10]; Cruz et al. (2003) [C03]; Chiu et al. (2006) [C06]; Cruz et al. (2007) [C07]; Dahn et al. (2002) [D02]; Deacon \& Hambly (2007) [D07]; Delfosse et al. (1997) [D97]; Dupuy \& Liu (2012)[D12]; Folkes et al. (2007)[F07]; Faherty et al. (2012) [F12]; Fan et al. (2000) [F00]; Gizis et al. (2000) [Giz00]; Gizis (2002) [Giz02]; Kendall et al. (2004) [Ken04]; Kendall et al. (2007) [K07]; Kirkpatrick et al. (1999) [K99]; Kirkpatrick et al. (2008) [K08]; Knapp et al. (2004) [K04]; Reid et al. (2000) [R00]; Reid et al. (2006a) [R06]; Reid et al. (2006b) [RL06]; Reid et al. (2008) [R08]; Smart et al. (2013) [S13]; Vrba et al. (2004) [V04]. 
P. A. Wilson et al.: The brown dwarf atmosphere monitoring (BAM) project. I.

Table 2. T dwarf sample.

\begin{tabular}{|c|c|c|c|c|c|c|}
\hline Target name & $\begin{array}{c}\text { Spectral } \\
\text { type }\end{array}$ & $\begin{array}{c}\text { Parallax } \\
(\mathrm{mas})\end{array}$ & $\begin{array}{c}J_{2 \mathrm{MASS}} \\
(\mathrm{mag})\end{array}$ & $\begin{array}{l}\text { Binary/ } \\
\text { Single }\end{array}$ & Instrument & References \\
\hline 2MASS J00345157+0523050 & T6.5 & $105.4 \pm 7.5$ & $15.535 \pm 0.045$ & & & B04, B06, F12 \\
\hline 2MASS J00501994-3322402 & $\mathrm{T} 7$ & $94.6 \pm 2.4$ & $15.928 \pm 0.07$ & & & T05, B06, D12 \\
\hline SIMP J013656.5+093347.3 & $\mathrm{T} 2.5$ & & $13.455 \pm 0.03$ & & & A06 \\
\hline 2MASS J03480772-6022270 & $\mathrm{T} 7$ & & $15.318 \pm 0.05$ & S & HST imaging & B03, BK06 \\
\hline 2MASS J04151954-0935066 & T8 & $175.2 \pm 1.7$ & $15.695 \pm 0.058$ & $\mathrm{~S}$ & HST imaging & B02, B06, V04, BK06 \\
\hline SDSS J042348.56-041403.5 & T0 & $72.1 \pm 1.1$ & $14.465 \pm 0.027$ & B (160 mas) & HST imaging & $\mathrm{G} 02, \mathrm{~B} 06, \mathrm{~V} 04, \mathrm{~B} 05$ \\
\hline 2MASS J05103520-4208140 & T5 & & $16.222 \pm 0.087$ & & & L07 \\
\hline 2MASS J05160945-0445499 & T5.5 & $44.5 \pm 6.5$ & $15.984 \pm 0.079$ & $\mathrm{~S}$ & HST imaging & B03, F12, B06, BK06 \\
\hline 2MASS J05591914-1404488 & $\mathrm{T} 4.5$ & $96.6 \pm 1$ & $13.802 \pm 0.024$ & $\mathrm{~S}$ & HST imaging & $\mathrm{B} 00, \mathrm{~B} 06, \mathrm{D} 02, \mathrm{BK} 03$ \\
\hline 2MASS J07290002-3954043 & $\mathrm{T} 8$ & $126.3 \pm 8.3$ & $15.92 \pm 0.077$ & & & L07, F12 \\
\hline DENIS J081730.0-615520 & T6 & $203 \pm 13$ & $13.613 \pm 0.024$ & & & A10 \\
\hline 2MASS J09393548-2448279 & T8 & $187.3 \pm 4.6$ & $15.98 \pm 0.106$ & & & T05, В06, В08 \\
\hline 2MASS J09490860-1545485 & $\mathrm{T} 2$ & $55.3 \pm 6.6$ & $16.149 \pm 0.117$ & ?B (weak candidate) & SpeX spectra & $\mathrm{T} 05, \mathrm{~F} 12, \mathrm{~B} 06, \mathrm{BC} 10$ \\
\hline 2MASS J10073369-4555147 & T5 & $71.0 \pm 5.2$ & $15.653 \pm 0.068$ & & & L07, F12 \\
\hline 2MASS J10210969-0304197 & T3 & $29.9 \pm 1.3$ & $16.253 \pm 0.091$ & B (172 mas) & HST imaging & L00, B06, T03, BK06 \\
\hline 2MASS J11145133-2618235 & $\mathrm{T} 7.5$ & $179.2 \pm 1.4$ & $15.858 \pm 0.083$ & & & T05, B06, D12 \\
\hline 2MASS J12074717+0244249 & T0 & $44.5 \pm 12.2$ & $15.58 \pm 0.071$ & ?B (weak candidate) & SpeX spectra & $\mathrm{H} 02, \mathrm{~F} 12, \mathrm{~B} 06, \mathrm{BC} 10$ \\
\hline 2MASS J12171110-0311131 & $\mathrm{T} 7.5$ & $90.8 \pm 2.2$ & $15.86 \pm 0.061$ & $\mathrm{~s}$ & HST imaging & B99, B06, T03, BK06 \\
\hline 2MASS J12255432-2739466 & T6 & $75.1 \pm 2.5$ & $15.26 \pm 0.047$ & B (282 mas) & HST imaging & B99, B06, Т03, ВK03 \\
\hline 2MASS J12314753+0847331 & T5.5 & & $15.57 \pm 0.072$ & & & B04, B06 \\
\hline 2MASS J12545393-0122474 & $\mathrm{T} 2$ & $84.9 \pm 1.9$ & $14.891 \pm 0.035$ & $\mathrm{~S}$ & HST imaging & L00, B06, D02, BK06 \\
\hline 2MASS J14044941-3159329 & $\mathrm{T} 2.5$ & $42.1 \pm 1.1$ & $15.577 \pm 0.062$ & B (130 mas) & AO imaging & L07, D12, L08 \\
\hline CFBDS J151114+060742 & $\mathrm{T} 2$ & $36.7 \pm 6.4$ & $16.016 \pm 0.079$ & ?B (strong candidate) & SpeX spectra & $\mathrm{A} 11, \mathrm{~F} 12, \mathrm{BC} 10$ \\
\hline 2MASS J15210327+0131426 & $\mathrm{T} 2$ & $41.3 \pm 7.2$ & $16.399 \pm 0.102$ & $\mathrm{~S}$ & SpeX spectra & $\mathrm{K} 04, \mathrm{~F} 12, \mathrm{~B} 06, \mathrm{BC} 10$ \\
\hline 2MASS J15344984-2952274 & T5.5 & $62.4 \pm 1.3$ & $14.9 \pm 0.054$ & B (65 mas) & HST imaging & B02, B06, T03, ВK03 \\
\hline 2MASS J15462718-3325111 & T5.5 & $88 \pm 1.9$ & $15.631 \pm 0.051$ & $\mathrm{~S}$ & HST imaging & $\mathrm{B} 02, \mathrm{~B} 06, \mathrm{~T} 03, \mathrm{BK} 03$ \\
\hline 2MASS J15530228+1532369 & $\mathrm{T} 7$ & $75.1 \pm 0.9$ & $15.825 \pm 0.071$ & B (349 mas) & HST imaging & B02, B06, D12, BK06 \\
\hline 2MASS J16241436+0029158 & T6 & $90.9 \pm 1.2$ & $15.494 \pm 0.054$ & $\mathrm{~S}$ & HST imaging & S99, B06, T03, BK06 \\
\hline 2MASS J18283572-4849046 & T5.5 & $83.7 \pm 7.7$ & $15.175 \pm 0.056$ & & & B04, F12, B06 \\
\hline SDSS J204749.61-071818.3 & T0 & $49.9 \pm 7.9$ & $16.85 \pm 0.04^{a}$ & $\mathrm{~S}$ & SpeX spectra & $\mathrm{K} 04, \mathrm{~F} 12, \mathrm{~B} 06, \mathrm{BC} 10$ \\
\hline 2MASS J20523515-1609308 & $\mathrm{T} 1$ & $33.9 \pm 0.8$ & $16.334 \pm 0.118$ & $\begin{array}{l}\text { ?B (weak candidate) } \\
\text { B (100.9 mas) }\end{array}$ & $\begin{array}{l}\text { SpeX spectra } \\
\text { AO imaging }\end{array}$ & $\begin{array}{c}\text { C06, D12, BC10 } \\
\text { S11 }\end{array}$ \\
\hline 2MASS J21392676+0220226 & $\mathrm{T} 1.5$ & $101.5 \pm 2.0$ & $15.264 \pm 0.049$ & ?B (strong candidate) & SpeX spectra & $\mathrm{R} 08, \mathrm{~B} 06, \mathrm{~S} 13, \mathrm{BC} 10$ \\
\hline 2MASS J21513839-4853542 & T4 & $50.4 \pm 6.7$ & $15.73 \pm 0.074$ & & & $\mathrm{E} 05, \mathrm{~F} 12, \mathrm{~B} 06$ \\
\hline 2MASS J22282889-4310262 & T6.5 & $94.0 \pm 7.0$ & $15.662 \pm 0.073$ & $\mathrm{~S}$ & HST imaging & B03, F12, B06, BK06 \\
\hline ULAS J232123.79+135454.9 & $\mathrm{T} 7.5$ & & $17.04 \pm 0.04^{a}$ & & & $\mathrm{~S} 10, \mathrm{~B} 10$ \\
\hline 2MASS J23312378-4718274 & T5 & & $15.659 \pm 0.068$ & & & B04, B06 \\
\hline 2MASS J23565477-1553111 & T5.5 & $57.9 \pm 3.5$ & $15.824 \pm 0.057$ & $\mathrm{~S}$ & HST imaging & В02, B06, S13, ВK03 \\
\hline
\end{tabular}

Notes. ${ }^{(a)}$ MKO to 2MASS conversion using transformations from Stephens \& Leggett (2004).

References. Albert et al. (2011) [A11]; Artigau et al. (2006) [A06]; Artigau et al. (2010) [A10]; Berger (2006) [B06]; Bouy et al. (2003) [B03]; Burgasser et al. (1999) [B99]; Burgasser et al. (2000) [B00]; Burgasser et al. (2003) [B02]; Burgasser et al. (2003) [BK03]; Burgasser et al. (2004) [B04]; Burgasser et al. (2005) [B05]; Burgasser et al. (2006) [BK06]; Burgasser et al. (2008) [B08]; Burgasser et al. (2010) [BC10]; Burningham et al. (2010) [B10]; Chiu et al. (2006) [C06]; Dahn et al. (2002) [D02]; Dupuy \& Liu (2012) [D12]; Ellis et al. (2005) [E05]; Faherty et al. (2012) [F12]; Gelino et al. (2002) [G02]; Hawley et al. (2002) [H02]; Knapp et al. (2004) [K04]; Leggett et al. (2000) [L00]; Looper et al. (2007) [L07]; Looper et al. (2008) [L08]; Reid et al. (2008) [R08]; Scholz et al. (2003) [S03]; Scholz et al. (2003) [S10]; Stumpf et al. (2011) [S11]; Smart et al. (2013) [S13]; Strauss et al. (1999) [S99]; Tinney et al. (2003) [T03]; Tinney et al. (2005) [T05]; Vrba et al. (2004) [V04]. 
A\&A 566, A111 (2014)

Table 4. Limits on constant targets in this survey.

\begin{tabular}{|c|c|c|c|c|c|c|c|c|}
\hline Object & Spectral type & Obs. dur. [h] & Refs. & DOF & $\chi_{v}^{2}$ & $\tilde{\eta}$ & $Q(\%)$ & $p$-value $(\%)$ \\
\hline 2MASS J00165953-4056541 & L3.5 & 3.43 & 6 & 8 & 0.9 & 0.8 & 0.54 & 51.1 \\
\hline 2MASS J00184613-6356122 & L2 & 3.43 & 7 & 8 & 1.1 & 1.0 & 0.42 & 35.4 \\
\hline 2MASS J00345157+0523050 & T6.5 & 2.98 & 7 & 7 & 0.9 & 1.1 & 0.53 & 49.1 \\
\hline 2MASS J01282664-5545343 & L1 & 2.66 & 5 & 5 & 0.5 & 2.1 & 0.61 & 77.7 \\
\hline 2MASS J02284355-6325052 & L0 & 2.84 & 3 & 10 & 0.8 & 1.0 & 0.42 & 62.7 \\
\hline 2MASS J02572581-3105523 & L8 & 3.33 & 7 & 5 & 0.3 & 1.2 & 0.48 & 90.2 \\
\hline 2MASS J03185403-3421292 & L7 & 1.97 & 5 & 4 & 1.9 & 1.8 & 0.99 & 10.1 \\
\hline 2MASS J03400942-6724051 & L7 & 2.61 & 6 & 5 & 0.2 & 1.1 & 0.75 & 96.4 \\
\hline 2MASS J04070752+1546457 & L3.5 & 3.19 & 7 & 7 & 0.7 & 0.7 & 0.43 & 68.4 \\
\hline 2MASS J04151954-0935066 & $\mathrm{T} 8$ & 3.29 & 4 & 7 & 0.3 & 0.6 & 0.83 & 95.8 \\
\hline SDSS J042348.56-041403.5 & T0 & 2.62 & 8 & 5 & 0.4 & 0.7 & 0.51 & 86.2 \\
\hline 2MASS J04455387-3048204 & $\mathrm{L} 2$ & 4.55 & 7 & 9 & 0.7 & 0.7 & 0.36 & 68.7 \\
\hline 2MASS J05103520-4208140 & T5 & 2.74 & 6 & 6 & 0.5 & 0.6 & 0.66 & 84.2 \\
\hline 2MASS J05160945-0445499 & T5.5 & 3.11 & 4 & 7 & 1.1 & 1.1 & 0.88 & 38.5 \\
\hline 2MASS J05233822-1403022 & L5 & 4.56 & 6 & 9 & 0.7 & 1.0 & 0.99 & 66.8 \\
\hline 2MASS J05591914-1404488 & $\mathrm{T} 4.5$ & 3.11 & 6 & 7 & 0.3 & 0.5 & 0.37 & 96.3 \\
\hline 2MASS J06244595-4521548 & L5 & 3.26 & 5 & 6 & 1.2 & 1.0 & 0.49 & 28.5 \\
\hline 2MASS J07290002-3954043 & $\mathrm{T} 8$ & 3.37 & 8 & 10 & 0.6 & 0.6 & 0.9 & 84.3 \\
\hline DENIS J081730.0-615520 & T6 & 3.48 & 8 & 13 & 0.8 & 0.7 & 0.68 & 64.5 \\
\hline 2MASS J09153413+0422045 & L7 & 3.33 & 6 & 7 & 0.9 & 0.8 & 0.55 & 50.1 \\
\hline 2MASS J09393548-2448279 & T8 & 3.06 & 8 & 9 & 1.0 & 0.8 & 0.98 & 46.5 \\
\hline 2MASS J09490860-1545485 & $\mathrm{T} 2$ & 3.16 & 8 & 6 & 1.7 & 1.3 & 0.67 & 11.0 \\
\hline 2MASS J10043929-3335189 & L4 & 3.14 & 8 & 11 & 1.4 & 1.0 & 0.99 & 17.6 \\
\hline 2MASS J10073369-4555147 & T5 & 3.41 & 8 & 13 & 0.3 & 0.5 & 1.62 & 99.5 \\
\hline 2MASS J10210969-0304197 & T3 & 3.17 & 7 & 6 & 0.7 & 0.6 & 0.88 & 64.1 \\
\hline 2MASS J11145133-2618235 & $\mathrm{T} 7.5$ & 2.90 & 5 & 5 & 0.4 & 1.0 & 1.0 & 86.3 \\
\hline 2MASS J11555389+0559577 & L7.5 & 2.87 & 5 & 7 & 0.6 & 1.2 & 0.74 & 79.3 \\
\hline 2MASS J12255432-2739466 & T6 & 2.85 & 7 & 5 & 0.4 & 0.3 & 0.67 & 86.9 \\
\hline 2MASS J12281523-1547342 & L6 & 3.39 & 7 & 10 & 1.0 & 1.1 & 0.5 & 42.5 \\
\hline 2MASS J12314753+0847331 & T5.5 & 2.56 & 5 & 5 & 0.3 & 1.6 & 1.63 & 89.6 \\
\hline 2MASS J12545393-0122474 & $\mathrm{T} 2$ & 3.17 & 7 & 9 & 1.0 & 1.1 & 0.51 & 45.2 \\
\hline 2MASS J13262981-0038314 & L5.5 & 2.80 & 4 & 7 & 0.9 & 0.9 & 1.17 & 50.8 \\
\hline 2MASS J14044941-3159329 & $\mathrm{T} 2.5$ & 3.01 & 8 & 8.5 & 0.4 & 0.6 & 0.82 & 89.4 \\
\hline 2MASS J15074769-1627386 & L5.5 & 4.16 & 7 & 15 & 0.4 & 0.6 & 1.21 & 98.5 \\
\hline CFBDS J151114+060742 & $\mathrm{T} 2.0$ & 3.85 & 7 & 11 & 0.8 & 0.6 & 0.8 & 64.1 \\
\hline 2MASS J15210327+0131426 & $\mathrm{T} 2$ & 6.37 & 7 & 12 & 0.5 & 0.6 & 0.9 & 93.6 \\
\hline 2MASS J15344984-2952274 & T5.5 & 3.88 & 8 & 8 & 0.5 & 0.6 & 0.62 & 85.3 \\
\hline 2MASS J15462718-3325111 & T5.5 & 3.50 & 4 & 7 & 0.4 & 0.5 & 1.45 & 93.3 \\
\hline 2MASS J15530228+1532369 & $\mathrm{T} 7$ & 3.82 & 7 & 8 & 2.7 & 1.0 & 0.72 & 0.7 \\
\hline 2MASS J16241436+0029158 & T6 & 3.21 & 8 & 8 & 0.3 & 0.4 & 0.66 & 95.6 \\
\hline 2MASS J16322911+1904407 & L8 & 3.89 & 4 & 14 & 1.1 & 1.0 & 1.76 & 36.4 \\
\hline 2MASS J18283572-4849046 & T5.5 & 3.06 & 8 & 7 & 0.4 & 0.5 & 0.5 & 88.7 \\
\hline 2MASS J19360187-5502322 & L5 & 3.21 & 7 & 6 & 0.4 & 0.5 & 0.4 & 86.9 \\
\hline SDSS J204317.69-155103.4 & L9.0 & 3.03 & 8 & 6 & 1.4 & 1.0 & 1.14 & 22.5 \\
\hline SDSS J204749.61-071818.3 & T0.0 & 2.68 & 8 & 5 & 1.6 & 1.2 & 1.16 & 15.8 \\
\hline 2MASS J20523515-1609308 & $\mathrm{T} 1$ & 3.08 & 8 & 7 & 0.8 & 0.8 & 0.7 & 62.9 \\
\hline 2MASS J21513839-4853542 & $\mathrm{T} 4$ & 3.08 & 8 & 7 & 0.8 & 0.7 & 0.51 & 63.0 \\
\hline 2MASS J22521073-1730134 & L7.5 & 2.34 & 5 & 5 & 0.8 & 1.3 & 0.65 & 58.9 \\
\hline ULAS J232123.79+135454.9 & $\mathrm{T} 7.5$ & 2.94 & 8 & 7 & 0.3 & 0.6 & 0.9 & 94.9 \\
\hline 2MASS J23224684-3133231 & L0 & 2.69 & 6 & 4 & 1.1 & 0.9 & 0.49 & 37.8 \\
\hline 2MASS J23312378-4718274 & T5 & 2.63 & 5 & 6 & 1.7 & 1.1 & 1.13 & 12.0 \\
\hline 2MASS J23565477-1553111 & T6 & 2.98 & 5 & 7 & 3.5 & 0.6 & 0.64 & 0.1 \\
\hline
\end{tabular}

